\title{
The resolution of an entropy puzzle for 4D non-BPS black holes
}

\author{
Nabamita Banerjee, ${ }^{a}$ Sukruti Bansal ${ }^{a, b}$ and Ivano Lodato ${ }^{a}$ \\ ${ }^{a}$ Department of Physics, IISER Pune, \\ Homi Bhabha Road, Pashan, Pune, India \\ ${ }^{b}$ Dipartimento di Fisica e Astronomia 'G. Galilei', Università di Padova, \\ and INFN, Sezione di Padova, \\ Via Marzolo 8, I-35131 Padova, Italy \\ E-mail: nabamita@iiserpune.ac.in, sukruti.bansal@pd.infn.it, \\ ivano@iiserpune.ac.in
}

ABstRACT: We show the equality between macroscopic and microscopic (statistical) black hole entropy for a class of four dimensional non-supersymmetric black holes in $\mathcal{N}=2$ supergravity theory, up to the first subleading order in their charges. This solves a long standing entropy puzzle for this class of black holes. The macroscopic entropy has been computed in the presence of a newly derived higher-derivative supersymmetric invariant of [1], connected to the five dimensional supersymmetric Weyl squared Lagrangian. Microscopically, the crucial role in obtaining the equivalence is played by the anomalous gauge gravitational Chern-Simons term.

Keywords: Black Holes in String Theory, Chern-Simons Theories, Supergravity Models ARXIV EPRINT: 1602.05326 


\section{Contents}

1 Introduction 1

$2 \mathcal{N}=2$ superconformal gravity in $4 \mathrm{D}$ setting $\quad 4$

$\begin{array}{ll}2.1 & \text { The background and the auxiliary field }\end{array}$

2.2 The entropy function 8

3 Entropy of 5D/4D (non-)BPS black holes 9

3.1 Statistical entropy of (non-)BPS black holes in 5D: a sketch 10

$\begin{array}{lll}3.2 & 5 \mathrm{D} \text { vs } 4 \mathrm{D} \text { theories and solutions } & 14\end{array}$

$\begin{array}{lll}3.3 & \text { Entropy of (non-)BPS black holes } & 15\end{array}$

$\begin{array}{lll}4 & \text { Results and conclusions } & 17\end{array}$

$\begin{array}{ll}\text { A Conventions and useful identities } & 19\end{array}$

B Superconformal mutiplets - (covariant) Weyl, chiral, vector multiplets 20

\section{Introduction}

Black Holes are thermodynamic objects and can be described in terms of thermodynamic quantities like temperature and entropy. These properties are difficult to understand at the microscopic level using statistical mechanics as we need a fundamental theory of quantum gravity to give the microscopic description of black holes. String theory, the most promising candidate of quantum gravity, has made a lot of progress in this regard. The observation that the thermodynamical properties of black holes can be studied and quantitatively computed with statistical methods in string theory has brought upon an overwhelming amount of research in the field. Supergravity theory, a low energy limit of string theory, has black holes as classical solutions. Hence, by linking the black holes solutions of supergravity to the states in string theory (carrying the same charges), one can hope to relate the (macroscopic) entropy of a black hole to the logarithm of the degeneracies of its (stringy) microstates.

The initial perfect laboratory on which this link was studied was that of BPS black holes and BPS saturated stringy states. In fact, the computation of statistical or microscopic black hole entropy in string theory makes use of the supersymmetry invariance of the background, which highly restricts the possible interactions between the constituent strings and branes, and it also protects certain quantities from the effect of couplings, thus allowing the entropy computed at zero coupling to be valid even in the presence of strong couplings. Therefore, the entropy of BPS black holes has been studied quite extensively 
using the statistical counting approach. Although the results obtained for the entropy of BPS black holes are highly satisfactory and have led to a deeper understanding of both black hole physics and statistical string theory, it would be much more insightful to have the equivalence between the two systems worked out in the more general context of non-BPS black holes and non-BPS stringy states. Unfortunately, on the string side of this equivalence, not many advancements have been made in a general non-supersymmetric context. Nevertheless, precise results exist for certain specific cases, and in this paper we will focus on one of them. We shall study the subleading corrections to the macroscopic entropy of extremal non-supersymmetric black holes in $\mathcal{N}=2$ supergravity in four dimensions and show its relation with the microscopic result.

The thermodynamical or macroscopic entropy of black holes can be worked out irrespective of any special symmetry. It is well known that the classical entropy of a black hole, given by Bekenstein-Hawking (BH) area law, corresponds to the area of the black hole's event horizon. Subleading corrections to BH formula, in the semiclassical "thermodynamical limit" of large charges, can be obtained by a method engineered by Wald [2] long ago. In this paper we use another, different but equivalent, method, known as the "entropy function formalism", given by Sen [3], which is well-suited to compute the macroscopic entropy of extremal black holes, and sub-leading corrections thereof.

The computation of semiclassical microscopic entropy of black holes, which is the logarithm of the degeneracy of states in string theory in the limit of large black hole charges, hinges strongly on supersymmetry of the theory. This entropy coincides at leading order to the statistical entropy, which we will compute using the partition function of $\mathrm{AdS}_{3}$ spacetimes. ${ }^{1}$ The final result is given by the Cardy formula

$$
S_{\mathrm{BH}}=2 \pi\left(\sqrt{\frac{c_{L} h_{L}}{6}}+\sqrt{\frac{c_{R} h_{R}}{6}}\right),
$$

where $c_{L}$ and $c_{R}$ are the left and right-handed central charges respectively in conformal field theory, and $h_{L}$ and $h_{R}$ are the left and right-handed zero modes of Virasoro generators respectively. Note that, since the goal is to compare the statistical result with the macroscopic one, the stringy states must correspond to a black hole configuration.

There are specific examples of string configurations which allow an exact counting of states even in the absence of supersymmetry [4-6]. Their approach is based on a more basic/primitive form of the AdS/CFT correspondence [7] as we will explain later on.

The final result for the microscopic entropy of a large class of five dimensional nonsupersymmetric black holes in $\mathcal{N}=2$ supergravity with subleading corrections as obtained in $[4-6]$ is given by

$$
S_{\text {non-BPS }}^{\text {micro }}=2 \pi \sqrt{q_{0}\left(d_{A B C} p^{A} p^{B} p^{C}+128 d_{A} p^{A}\right)}
$$

where $q_{0}$ is the electric charge of the black hole, $p^{A}$ 's are the magnetic charges, and $d_{A B C}$ and $d_{A}$ are constant factors appearing in the prepotential of the Lagrangian of the black hole

\footnotetext{
${ }^{1}$ We will refer henceforth, abusing the terminology, to microscopic and statistical entropy interchangeably, although strictly speaking the two entropies are computed in opposite regimes and using very different methods.
} 
solution. ${ }^{2}$ The computation of the macroscopic counterpart of the statistically calculated entropy (1.1) was attempted in [8] and the following result was obtained:

$$
S_{\text {non-BPS }}^{\text {macro }}=2 \pi \sqrt{q_{0}\left(d_{A B C} p^{A} p^{B} p^{C}\right)}\left(1+\frac{40 d_{A} p^{A}}{d_{A B C} p^{A} p^{B} p^{C}}\right) .
$$

Evidently, this does not correspond to a first order expansion of (1.2) in the limit of large charges. The most likely reason behind this mismatch, as proposed in [8], is connected to the form of the higher derivative interaction terms. To be precise, the Weyl squared invariant of superconformal gravity, that was considered in [8], might not be enough to describe all the subleading contributions for a non-BPS solution. This directed us towards the possibility of certain (supersymmetric) higher derivative invariants missing in the four dimensional Lagrangian, that could be connected to five dimensional theories considered in $[5,6]$, and hence readily affect the macroscopic results.

Recently, a new class of higher derivative invariants was built in the context of four dimensional superconformal gravity [1]. The most important property of this new class of invariants is its connection to the five dimensional supersymmetrization of the Weyl squared Lagrangian, which contains the gauge gravitational Chern-Simons term considered in $[5,6]$. This higher derivative invariant has also been shown to not contribute to the entropy of BPS black holes [9]. This was expected because the micro- and macroscopic entropy results for BPS black holes already matched [10-12] without considering these corrections. In this paper, we consider this new higher derivative invariant to compute its contribution to the entropy of the non-BPS black hole solutions. The final entropy we obtain is given as,

$$
S_{\text {non-BPS }}^{\text {macro }}=2 \pi \sqrt{q_{0}\left(d_{A B C} p^{A} p^{B} p^{C}\right)}\left(1+\frac{64 d_{A} p^{A}}{d_{A B C} p^{A} p^{B} p^{C}}\right)
$$

and it exactly matches the first order expansion of statistical entropy in the limit of large charges (1.2), as predicted in [5, 6]! Hence, we have successfully moved one step further in describing black hole thermodynamics in the context of string theory via the more general case of non-BPS black holes in supergravity.

In the following we will closely follow [8], focusing on a general class of theories, the STU models. The paper is organized as follows: in section 2 we present the $4 \mathrm{D}$ theory in its prepotential formulation and add a new class of supersymmetric higher derivative invariants, recently discovered and analyzed in $[1,9]$. The new higher derivative invariant was the missing ingredient in the previous calculation worked out in [8] and it is the key to the resolution of the puzzle. In section 3 we give an outline of the microscopic entropy computation for a specific class of black holes, and make contact with the lower dimensional theory and solutions in four dimensions. Finally in section 4 we conclude with the results and open questions. Our conventions and the details of the $4 \mathrm{D} \mathcal{N}=2$ superconformal theory are presented in the two appendices at the end of this paper.

\footnotetext{
${ }^{2}$ Their physical interpretation will be given in section 3.1 .
} 


\section{$2 \mathcal{N}=2$ superconformal gravity in $4 \mathrm{D}$ setting}

We want to study the subleading correction to the entropy of non-BPS black holes in four dimensional $\mathcal{N}=2$ supergravity. The theory we use describes the dynamics of four vector multiplets $X^{I}(I, J=0, \ldots, 3)$ coupled to conformal supergravity and a chiral background multiplet $\boldsymbol{A}$, which will soon be identified with a particular linear combination of the Weyl squared multiplet [13] and the TLog multiplet [1]. This, as shown in [14] and proven in [9], corresponds exactly to the dimensional reduction of the $5 \mathrm{D}$ higher derivative invariant containing the Weyl squared term and the gravitational Chern-Simons term $A \wedge R \wedge R$ [15].

The Lagrangian can be written in terms of a homogeneous function of degree $2, F$ $(A, B=1,2,3)$ :

$$
F\left(X^{I}, \mathcal{A}\right)=-d_{A B C} \frac{X^{A} X^{B} X^{C}}{X^{0}}-d_{A} \frac{X^{A}}{X^{0}} \mathcal{A}
$$

where $X^{I}$ and $\mathcal{A}$ are the lowest components of the vector and chiral multiplets of $\mathcal{N}=2$ theory respectively:

$$
\mathcal{X}^{I}=\left(X^{I}, \Omega_{i}^{I}, W_{\mu}^{I}, Y_{i j}^{I}\right), \quad \boldsymbol{A}=\left(\mathcal{A}, \boldsymbol{\psi}_{i}, \mathcal{B}_{i j}, \mathcal{F}_{a b}^{-}, \boldsymbol{\Lambda}_{i}, \mathcal{C}\right)
$$

We refer to appendix B for a more detailed discussion on the supersymmetry transformation rules of these multiplets. For now it suffices to say that they are representations of the full superconformal algebra $\mathrm{SU}(2,2 \mid 2)$, which, in this particular example, corresponds to the algebra of the symmetries of the Lagrangian. ${ }^{3}$ For simplicity, we will focus only on the bosonic sector of the action, which reads ${ }^{4}$ (refer to the appendices A and B for the definitions of the fields and derivatives used in the following):

$$
\begin{aligned}
8 \pi \mathcal{L}= & \mathrm{i}\left(X^{I} \bar{F}_{I}-\bar{X}^{I} F_{I}\right)\left(\frac{1}{6} R-D\right)+\left[\mathrm{i} \mathcal{D}_{a} F_{I} \mathcal{D}^{a} \bar{X}^{I}\right. \\
& +\frac{\mathrm{i}}{4} F_{I J}\left(F_{a b}^{-I}-\frac{1}{4} \bar{X}^{I} T_{a b}^{-}\right)\left(F^{-J a b}-\frac{1}{4} \bar{X}^{J} T^{-a b}\right) \\
& +\frac{\mathrm{i}}{8} \bar{F}_{I}\left(F_{a b}^{-I}-\frac{1}{4} \bar{X}^{I} T_{a b}^{-}\right) T^{-a b}-\frac{\mathrm{i}}{8} F_{I J} Y_{i j}^{I} Y^{J i j}+\frac{\mathrm{i}}{32} \bar{F}\left(T_{a b}^{-}\right)^{2} \\
& +\frac{\mathrm{i}}{2} F_{\mathcal{A}} \mathcal{C}-\frac{\mathrm{i}}{8} F_{\mathcal{A} \mathcal{A}}\left(\mathcal{B}_{i j} \mathcal{B}^{i j}-2 \mathcal{F}_{a b}^{-} \mathcal{F}^{-a b}\right) \\
& \left.+\frac{\mathrm{i}}{2} \mathcal{F}^{-a b} F_{\mathcal{A} I}\left(F_{a b}^{-I}-\frac{1}{4} \bar{X}^{I} T_{a b}^{-}\right)-\frac{\mathrm{i}}{4} \mathcal{B}_{i j} F_{\mathcal{A} I} Y^{I i j}+h . c .\right] \\
& +\mathrm{i}\left(X^{I} \bar{F}_{I}-\bar{X}^{I} F_{I}\right)\left(\mathcal{D}^{a} V_{a}-\frac{1}{2} V^{a} V_{a}-\frac{1}{4}\left|M_{i j}\right|^{2}\right. \\
& \left.+\left(\partial^{\mu} \Phi_{\alpha}^{i}+\frac{1}{2} \mathcal{V}^{i \mu} \Phi_{\alpha}^{j}\right)\left(\partial_{\mu} \Phi_{i}^{\alpha}+\frac{1}{2} \mathcal{V}_{i \mu}^{k} \Phi_{k}^{\alpha}\right)\right)
\end{aligned}
$$

\footnotetext{
${ }^{3}$ R-symmetry is not necessarily a symmetry of the action.

${ }^{4}$ Our conventions differ from [8] by a minus sign in the Riemann tensor and we write down the explicit dependence on $\mathrm{D}$ in the action.
} 
where the following definitions have been used:

$$
\begin{aligned}
F_{\mu \nu}^{I} & =2 \partial_{[\mu} W^{I}{ }_{\nu]}, & & T_{a b}^{-}=T_{a b}{ }^{i j} \varepsilon_{i j}, \\
F_{I} & =\partial_{X^{I}} F, & & F_{\mathcal{A}}=\partial_{\mathcal{A}} F, \ldots .
\end{aligned}
$$

In Lagrangian (2.3), $V_{a}$ is a vector field, $M_{i j}$ is an $\mathrm{SU}(2)$ triplet scalar field and $\Phi_{i}^{\alpha}$ is an $\mathrm{SU}(2)$ matrix valued scalar field. The indices $i, j(=1,2)$ and $\alpha(=1,2)$ used here, label the fundamental representation of gauged and global $\mathrm{SU}(2)$ respectively.

We couple the theory to a non-linear multiplet whose gauge field $V_{\mu}$ is subject to the constraint:

$$
D^{a} V_{a}-\frac{1}{2} V^{a} V_{a}-\frac{1}{4}\left|M_{i j}\right|^{2}+\left(\partial^{\mu} \Phi_{\alpha}^{i}+\frac{1}{2} \mathcal{V}_{j}^{i \mu} \Phi_{\alpha}^{j}\right)\left(\partial_{\mu} \Phi_{i}^{\alpha}+\frac{1}{2} \mathcal{V}_{i \mu}^{k} \Phi_{k}^{\alpha}\right)-3 D=0
$$

which reduces the number of its independent degrees of freedom from four to three, balancing the number of independent bosonic and fermionic fields. More importantly it allows to fix the value of the auxiliary field $D$ in terms of the Ricci scalar $R$. Note that $V_{\mu}$ transforms under K-boosts, so its covariant derivative can be written as $D_{a} V^{a}=\mathcal{D}_{a} V^{a}-2 f_{a}^{a}=$ $\mathcal{D}_{a} V^{a}-\frac{1}{3} R+2 D$.

We are now ready to identify the chiral background with the two higher derivative invariants that come from the dimensional reduction of the $R^{2}$ term in $5 \mathrm{D} \mathcal{N}=2$ supergravity, the Weyl squared invariant and the TLog multiplet, i.e [9]

$$
\mathcal{A}=\left(T_{a b}^{-}\right)^{2}-\left.\frac{32}{3} A\right|_{\mathbb{T}\left(\ln \bar{X}^{0}\right)} .
$$

Although the Weyl squared invariant depends only on conformal supergravity curvatures and auxiliary fields, the TLog multiplet, as it comes from the dimensional reduction from $5 \mathrm{D}$, depends explicitly on the compensating vector multiplet $\mathcal{X}^{0}$. Of course, it would seem logical (but nevertheless incorrect) to consider the dependence of $\boldsymbol{A}$ on $\mathcal{X}^{0}$, while taking the derivatives of the prepotential w.r.t. the scalar fields $X^{I}$. The multiplets at this stage must be considered elementary (independent). This means, for instance, that the derivative of the prepotential w.r.t. to $X_{0}$ is simply given by $F_{0}=d_{A} \frac{X^{A}}{X_{0}^{2}} \mathcal{A}$. Once the supersymmetric Lagrangian has been obtained, the components of $\boldsymbol{A}$ can be traded with their composite expressions. Neglecting all the fermions, these components read (the indices $a, b=0, \ldots, 3$ are flat or tangent space indices)

$$
\begin{aligned}
\mathcal{A}= & \left(T_{a b}^{-}\right)^{2}-\frac{32}{3}\left(-2 \frac{\square_{\mathrm{c}} X^{0}}{\bar{X}^{0}}-\frac{1}{4} \frac{\mathcal{F}_{a b}^{-0} T^{-a b}}{\bar{X}^{0}}+\frac{1}{4\left(\bar{X}^{0}\right)^{2}}\left(Y^{0 i j} Y_{i j}^{0}-2 \mathcal{F}_{a b}^{+0} \mathcal{F}^{+0 a b}\right)\right), \\
\mathcal{B}_{i j}= & -16 \varepsilon_{k(i} R(\mathcal{V})^{k}{ }_{j) a b} T^{-a b}+\frac{64}{3}\left(\square_{\mathrm{c}}+3 D\right) \frac{Y^{i j}}{\bar{X}^{0}}+\frac{64}{3} \frac{\mathcal{F}_{a b}^{+0}}{\bar{X}^{0}} R(\mathcal{V})^{a b k}{ }_{i} \varepsilon_{j k}, \\
\mathcal{F}_{a b}^{-}= & -16 \mathcal{R}(M)_{c d}{ }^{a b} T^{-c d}-\frac{32}{3} \square_{c} \log \bar{X}^{0} T_{a b}^{-}+\frac{32}{3} R(\mathcal{V})^{-}{ }_{a b}{ }_{k}{ }_{k} \frac{Y^{0 j k}}{\bar{X}^{0}} \varepsilon_{i j}-\frac{2}{3} T_{a b}^{-} T_{c d}^{+} \frac{\mathcal{F}^{+0} \bar{X}^{0}}{\bar{X}^{0}} \\
& +\frac{32}{3}\left(\delta_{a}{ }^{[c} \delta_{b}{ }^{d]}-\frac{1}{2} \varepsilon_{a b}{ }^{c d}\right)\left[4 D_{c} D^{e} \frac{\mathcal{F}_{e d}^{+0}}{\bar{X}^{0}}+\left(D^{e} \log \bar{X}^{0} D_{c} T_{d e}^{-}+D_{c} \log \bar{X}^{0} D^{e} T_{e d}^{-}\right)\right. \\
& \left.-w D_{c} D^{e} T_{e d}^{-}\right]
\end{aligned}
$$




$$
\begin{aligned}
\mathcal{C}= & 64 \mathcal{R}(M)^{-c d}{ }_{a b} \mathcal{R}(M)_{c d}^{-a b}+32 R(\mathcal{V})^{-a b k}{ }_{l} R(\mathcal{V})_{a b k}^{-l}-16 T^{-a b} D_{a} D^{c} T_{c b}^{+} \\
& -\frac{128}{3}\left(\square_{c}+3 D\right) \square_{c} \log \bar{X}^{0}-64\left(D_{a} D\right) D^{a} \log \bar{X}^{0}+\frac{512}{3} D^{a}\left(R(D)_{a b}^{+} D^{b} \log \bar{X}^{0}\right) \\
& +\frac{16}{3} D^{a}\left(T_{a b}^{+} T^{-c b} D_{c} \log \bar{X}^{0}\right)+\frac{8}{3} D^{a}\left(T_{a b}^{+} T^{-c b}\right) D_{c} \log \bar{X}^{0} \\
& -\frac{16}{3} D_{a} D^{a}\left(T_{b c}^{+} \frac{\mathcal{F}^{+0 b c}}{\bar{X}^{0}}\right)-\frac{64}{3} D_{a}\left(D^{b} T_{b c}^{+} \frac{\mathcal{F}^{+0 a c}}{\bar{X}^{0}}+D^{b} \frac{\mathcal{F}_{b c}^{+0}}{\bar{X}^{0}} T^{+a c}\right) \\
& -\left.\frac{2}{3}\left(T_{a b}^{+}\right)^{2} A\right|_{\mathbb{T}\left(\log \bar{X}^{0}\right)}-\frac{32}{3} w\left\{-R(\mathcal{V})_{a b}^{+i}{ }_{j} R(\mathcal{V})^{a b+j}{ }_{i}-8 R(D)_{a b}^{+} R(D)^{a b+}\right. \\
& \left.-\frac{1}{2} D^{a} T_{a b}^{+} D_{c} T^{-c b}-\frac{1}{2} D^{a}\left(T_{a b}^{+} D_{c} T^{-c b}\right)\right\},
\end{aligned}
$$

where $\mathcal{F}_{a b}^{-0}=F_{a b}^{-0}-\frac{1}{4} \bar{X}^{0} T_{a b}^{-}$and we made use of the constraints relating the components of a chiral multiplet to those of a vector multiplet (see (B.13)). Since we have fixed the TLog multiplet to be a composite function of the vector multiplet $\mathcal{X}^{0}$, the parameter $w$ above is fixed to one [1].

\subsection{The background and the auxiliary field}

We want to study the entropy of extremal, non-BPS black holes. The near horizon geometry of an extremal black hole in four dimensions is described by $\mathrm{AdS}_{2} \times \mathrm{S}^{2}$ metric, given by

$$
d s^{2}=v_{1}\left(-r^{2} \mathrm{~d} t^{2}+\frac{\mathrm{d} r^{2}}{r^{2}}\right)+v_{2}\left(\mathrm{~d} \theta^{2}+\sin ^{2} \theta \mathrm{d} \phi^{2}\right) .
$$

Here $v_{1}$ and $v_{2}$ are the scaling parameters of the $\mathrm{AdS}_{2}$ and $\mathrm{S}^{2}$ spaces respectively. The invariance under the symmetry group $\mathrm{SO}(2,1) \times \mathrm{SO}(3)$ of the $\mathrm{AdS}_{2} \times \mathrm{S}^{2}$ metric must be satisfied by all field configurations. As an example, in flat space indices, the field strengths will have only two non-zero components, one along $(0,1)$ direction and the other along $(2,3)$ direction as,

$$
F_{01}^{I}=-\frac{e^{I}}{v_{1}}, \quad F_{23}^{I}=\frac{p^{I}}{v_{2}},
$$

where $e^{I}$ 's are the generalized electric fields, $p^{I}$ 's are the magnetic charges of the black hole. The electric charges are defined via the dual field strength:

$$
G_{I a b}^{-}=(-2 \mathrm{i}) \frac{\partial(8 \pi \mathcal{L})}{\partial F^{-I a b}}
$$

and are given by:

$$
\frac{q_{I}}{v_{2}}=G_{I 23}
$$

Analogously the auxiliary $T_{a b}^{ \pm}$tensor will have only two non-zero components, one on the subspace $(0,1)$ and the other in the subspace $(2,3)$,

$$
T_{01}^{-}=\mathrm{i} T_{23}^{-}=-z,
$$

with $z$ complex constant. Another important quantity, which is completely fixed by the symmetries of the background, is the modified Lorentz curvature $\mathcal{R}(M)_{a b}{ }^{c d}$. Here we 
present the non-zero components (from here onwards we take $R(A)=0$ for reasons that will be explained shortly),

$$
\begin{aligned}
& \mathcal{R}(M)_{\underline{a b}} \underline{\underline{c d}}=\left(D+\frac{1}{3} R\right) \delta_{\underline{a} \underline{b}} \underline{c d}, \\
& \mathcal{R}(M)_{\hat{a} \hat{b}}^{\hat{b} \hat{d}}=\left(D+\frac{1}{3} R\right) \delta_{\hat{a} \hat{b}}^{\hat{b} \hat{d}}, \\
& \mathcal{R}(M)_{\underline{a} \hat{b}} \hat{b}=\frac{1}{2}\left(D-\frac{1}{6} R\right) \delta_{\underline{a}} \underline{\underline{c}} \delta_{\hat{b}}^{\hat{b}},
\end{aligned}
$$

where the underlined and hatted indices span the $(t, r)$ and $(\theta, \phi)$ subspaces respectively. Now, by using the definition of $\mathcal{R}(M)_{a b}{ }^{c d}$ in terms of the Weyl tensor $C_{a b}{ }^{c d}$,

$$
\mathcal{R}(M)_{a b}{ }^{c d}=C_{a b}{ }^{c d}+D \delta_{[a}^{c} \delta_{b]}^{d},
$$

we obtain

$$
\mathcal{R}(M)_{a b}^{ \pm a b}=\frac{1}{2} \mathcal{R}(M)_{a b}^{a b}=3 D .
$$

There are many other (non-)dynamical fields attracted on the horizon, and in principle one would need to solve their equations of motion to find their constant values explicitly. For simplicity, we will consider the consistent truncation of the full field configuration, satisfying the near-horizon symmetries and describing (non-)BPS black holes in $\mathcal{N}=2$ supergravity, presented in [8]. In the following we shall sketch the truncation procedure, proving its consistency. Although for the purpose of this paper we need consistent solutions of the two derivative theory only, the truncation remains consistent even after the addition of higher derivative invariants.

Let us start from the auxiliary fields of the vector multiplet, in particular $Y_{i j}^{I}$. The (full) equations of motion for these four triplets are quadratic in the $Y^{I}$ 's, so a simple consistent solution of the dynamical equations is $Y_{i j}^{I}=0$. Next, we analyze the auxiliary gauge fields, the R-symmetry connections of the Weyl multiplet $A_{\mu}$ and $\mathcal{V}_{\mu}^{i}{ }_{j}$ (see appendix B). We first note that, if $A_{\mu}$ is constant, its curvature $R(A)=0$. This means that locally $A_{\mu}$ can be taken to be vanishing. Furthermore, the vanishing of the $\mathrm{U}(1)_{\mathrm{R}}$ curvature implies $R(D)=0$ (eq. (B.8)) which implies that also the dilatational gauge $b_{\mu}$ can also be taken to be locally vanishing. On the other hand when the $\mathrm{SU}(2)_{\mathrm{R}}$ connection $\mathcal{V}_{\mu}^{i}{ }_{j}$ is constant, the curvature $R(\mathcal{V})_{\mu \nu}^{i}{ }_{j} \sim \mathcal{V}_{\mu} \mathcal{V}_{\nu}$. It is easy to check by inspection that the (higher derivative) equations of motion for $\mathcal{V}_{\mu}^{i}{ }_{j}$ are quadratic or quartic in it, so they also admit the solution $\mathcal{V}_{\mu j}^{i}=0$. The fields of the compensating non-linear multiplet can be trivially fixed through their equations of motion. The $\mathrm{SU}(2)$ triplet $M_{i j}$ can be fixed to zero, and the field $\Phi_{\alpha}^{i}$, obeying the constraints $\Phi^{\dagger} \Phi=\nVdash$ and $\operatorname{det} \Phi=1$, can be fixed to the constant $\delta_{\alpha}^{i}$. The vector $V_{\mu}$ is constrained by eq. (2.5), and admits the simple solution $V_{\mu}=0$. This in turn leads to a constraint on the auxiliary field $D$, i.e. $D+\frac{1}{3} R=0$.

The full near-horizon configuration at the two derivative level is then:

$$
\begin{aligned}
T_{01}^{-} & =-z, & A_{\mu} & =\mathcal{V}_{\mu j}{ }^{i}=b_{\mu}=V_{\mu}=M_{i j}=0, \\
\Phi_{\alpha}^{i} & =\delta_{\alpha}^{i}, & D & =-\frac{1}{3} R .
\end{aligned}
$$


We want to point out that on this background all the fields are covariantly constant because the symmetries of the geometry allow for spin connections orthogonal to the field configurations. For e.g., one can check explicitly that $\mathcal{D}_{\mu} T_{a b}^{ \pm}=0$. Note that the addition of the TLog multiplet brings about terms linear in the $\mathrm{SU}(2)_{R}$ connection through the covariant derivative of the auxiliary field $Y_{i j}^{0}$ of the vector multiplet $\mathcal{X}^{0}$. As we pointed out before, however, the auxiliary fields $Y_{i j}^{I}=0$, and so the linear dependence on $\mathcal{V}_{\mu j}{ }^{i}$ is removed. An equivalent approach would have been to assume all the fields to be covariantly constant and find, as a solution, $A_{\mu}=b_{\mu}=\mathcal{V}_{\mu}{ }^{i}{ }_{j}=0$.

Finally we note that the auxiliary real scalar $D$ does not appear in the Lagrangian at the two derivative level, so in principle, it cannot be fixed. However, since it enters the nonlinear multiplet constraint, its most general consistent configuration is indeed $D=-\frac{1}{3} R$ even after the higher derivative corrections are considered. But, as will become clear shortly, we will not need the near-horizon field configurations beyond the leading two derivative order, to compute the first order subleading corrections to the entropy of the (non)-BPS black hole solutions.

\subsection{The entropy function}

In this section we set up the computation of the moduli and the entropy of a class of black holes with near-horizon geometry (2.8). We will use Sen's entropy function formalism for this purpose. The entropy function is given as

$$
\mathcal{E}\left(v_{1}, v_{2}, z, X^{I}, e^{I}, q_{I}, p^{I}\right)=2 \pi\left(-\frac{1}{2} e^{I} q_{I}-\int \mathrm{d} \theta \mathrm{d} \phi \sqrt{-\operatorname{det} g} \mathcal{L}\right) .
$$

This function need to be evaluated for the background described by (2.16). Hence $\mathcal{E}$ will be a function of the charges and various near horizon parameters. The entropy function formalism requires the entropy function to be extremized with respect to the near horizon parameters, i.e. obey the following extremization equations:

$$
\frac{\partial \mathcal{E}}{\partial v_{1,2}}=\frac{\partial \mathcal{E}}{\partial X^{I}}=\frac{\partial \mathcal{E}}{\partial e^{I}}=\frac{\partial \mathcal{E}}{\partial z}=0
$$

The values of the moduli will either remain unfixed, as is the case for the auxiliary field $D$ at the two derivative level, or will get fixed in terms of the electric and magnetic charges $q_{I}$ and $p^{I}$. The extremum value of $\mathcal{E}$ by definition gives us the entropy of the black hole, i.e.

$$
S_{\mathrm{BH}}=\left.\mathcal{E}\right|_{v_{1,2}, z, X^{I}, e^{I}}
$$

Before we proceed to adapt the entropy function formalism for our purpose, we want to show how it simplifies the computations while treating the higher derivative Lagrangians perturbatively. Consider the higher derivative perturbative effective actions to be specified by a small parameter $\delta$. In their presence, various near horizon parameters will get corrections proportional to $\delta$. To be precise, let $\Phi$ denotes all possible near horizon parameters $\left(v_{1}, v_{2}, z, X^{I}, e^{I}, \ldots\right)$. In presence of perturbative higher derivative interactions, the entropy function can be schematically written as $\mathcal{E}=\mathcal{E}_{0}+\delta \mathcal{E}_{1}$ and the perturbative solutions of the near horizon parameters as $\Phi=\Phi_{0}+\delta \cdot \Phi_{1}$. Here $\mathcal{E}_{0}$ is the entropy function for 
two derivative theory and $\Phi_{0}$ is the solution of the corresponding extremization equations. Finally, the value of the extremized entropy function to first order in $\delta$ looks like,

$$
\mathcal{E}(\Phi)=\mathcal{E}_{0}\left(\Phi_{0}+\delta \Phi_{1}\right)+\delta \cdot \mathcal{E}_{1}\left(\Phi_{0}\right)=\mathcal{E}_{0}\left(\Phi_{0}\right)+\delta \cdot \mathcal{E}_{1}\left(\Phi_{0}\right)
$$

The dependence on $\Phi_{1}$ will vanish identically by the leading order extremization condition. This means, to compute the first subleading correction to the entropy of black holes, using the entropy function formalism, we will need only the two derivative solutions for the near horizon parameters. Hence, as hinted above, for computing non-BPS black holes entropy accurate up to the first order in $\delta$, i.e. due to the first order effective corrections of the theory, we will only need the consistent solutions for near horizon parameters presented in (2.16).

Now, plugging the near horizon configuration of section 2.1 into the entropy function for our case, we obtain:

$$
\begin{aligned}
\mathcal{E} \equiv & -\pi q_{I} e^{I}-\pi g\left(v_{1}, v_{2}, z, X^{I}, e^{I}, p^{I}\right) \\
= & -\pi q_{I} e^{I}-\pi\left\{\mathrm{i}\left(v_{2}-v_{1}\right)\left(X^{I} \bar{F}_{I}-\bar{X}^{I} F_{I}\right)\right. \\
& -\left[\frac{\mathrm{i}}{4} v_{2} v_{1}^{-1} F_{I J}\left(e^{I}-\mathrm{i} \frac{v_{1}}{v_{2}} p^{I}-\frac{1}{2} \bar{X}^{I} z v_{1}\right)\left(e^{J}-\mathrm{i} \frac{v_{1}}{v_{2}} p^{J}-\frac{1}{2} \bar{X}^{J} z v_{1}\right)+\text { h.c. }\right] \\
& -\left[\frac{\mathrm{i}}{4} \bar{F}_{I} z v_{2}\left(e^{I}-\mathrm{i} \frac{v_{1}}{v_{2}} p^{I}-\frac{1}{2} \bar{X}^{I} z v_{1}\right)+\text { h.c. }\right]+\left[\frac{\mathrm{i}}{8} F \bar{z}^{2} v_{1} v_{2}+\text { h.c. }\right] \\
& +\left[32 \mathrm{i} F_{\mathcal{A}}\left(\frac{8}{3} \frac{v_{1}}{v_{2}}+\frac{8}{3} \frac{v_{2}}{v_{1}}-4+\frac{7}{192} v_{1} v_{2}|z|^{4}-\frac{1}{3}|z|^{2}\left(v_{1}+v_{2}\right)\right)+\text { h.c. }\right] \\
& +\left[\left.\frac{4 \mathrm{i}}{3} F_{\mathcal{A}} v_{1} v_{2} \bar{z}^{2} A\right|_{\mathbb{T}\left(\ln \bar{X}^{0}\right)}+\text { h.c. }\right]-\left[\frac{16 z}{3 v_{1}}\left(v_{1}-v_{2}\right) F_{\mathcal{A} I}\left(e^{I}-\mathrm{i} \frac{v_{1}}{v_{2}} p^{I}-\frac{1}{2} v_{1} z \bar{X}^{I}\right)+\text { h.c. }\right] \\
& -\left[\frac{32}{3} \frac{1}{\bar{X}^{0}} \frac{v_{2}}{v_{1}} F_{\mathcal{A} I}\left(e^{I}-\mathrm{i} \frac{v_{1}}{v_{2}} p^{I}-\frac{1}{2} v_{1} z \bar{X}^{I}\right)\left(e^{0}+\mathrm{i} \frac{v_{1}}{v_{2}} p^{0}-\frac{1}{2} v_{1} \bar{z} X^{0}\right)\right. \\
& \left.\left.\times\left(-\frac{1}{v_{1}}-\frac{1}{v_{2}}+\frac{1}{4}|z|^{2}\right)+\text { h.c. }\right]\right\} .
\end{aligned}
$$

In the next section we will focus on two particular classes of solutions: fully BPS and non-BPS black holes, and we will compute their entropy by extremizing the entropy function $\mathcal{E}$. Before doing so, we will review the microscopic/statistical entropy results, based on anomalies.

\section{Entropy of 5D/4D (non-)BPS black holes}

As mentioned earlier, although the computation of non-supersymmetric indices is still not well understood, it is possbile to obtain quantitative results for the statistical entropy of non-BPS black holes in very specific cases. In the following we will give a short summary of the results obtained by [4-6] for five-dimensional supergravity black holes, stressing the crucial ingredients. It is worth stressing that identical results were already presented in [10], but there the interest was focused on BPS solutions. We will also discuss the connection to four-dimensional black hole solutions of $\mathcal{N}=2$ supergravity, relevant to this work. 


\subsection{Statistical entropy of (non-)BPS black holes in 5D: a sketch}

Consider a near-horizon black brane configuration of the form $\mathrm{AdS}_{3} \times S^{2} \times X$, where $X$ is a Calabi-Yau three-fold, that arises from M-theory. ${ }^{5}$ After compactifying on $X$, we get the near-horizon solution of a five dimensional black hole, which, depending on the nearhorizon values of the matter and gravity fields, may or may not preserve supersymmetries. The theory can be further reduced over a two-sphere to obtain a solution of three dimensional supergravity, which includes the dimensionally reduced Chern-Simons form $A \wedge \mathrm{d} A$. A crucial point is that the left and right central charges of the CFT that lives at the boundary of $\mathrm{AdS}_{3}$ can now be computed from the anomalous terms that are connected to the Chern-Simons terms of the reduced 5D supergravity Lagrangian. The procedure requires supersymmetry of the theory, but not necessarily of the background. Hence we will obtain a result for non-BPS configurations as well. In this section we want to sketch the main results of the anomaly analysis, referring to $[4-6,10,16,17]$ and the references therein for more detailed discussions.

The first goal of this section is to obtain the Cardy formula for the entropy of black holes whose near-horizon configuration contains an $\mathrm{AdS}_{3}$ factor [7]. Note that there are certain advantages in considering an $\mathrm{AdS}_{3}$ near-horizon configuration instead of $\mathrm{AdS}_{2}$, since the spacetime symmetries of the former close into the infinite dimensional Virasoro algebra.

One way to compute the black hole entropy is to consider the Euclidean continuation of the solution. One then obtains the thermodynamics potential from which it is straightforward to compute the entropy. In Euclidean signature, the BTZ black hole is a solid torus having one contractible and one non-contractible cycle. We denote the coordinates along these cycles respectively as $t_{C}$ and $t_{N C}$. There is an entire family of solutions that can be derived from the Euclidean one by choosing to identify the time coordinate with a certain combination of $t_{N C}$ and $t_{C}$. For our purpose it is enough to consider just the two cases, $t_{N C} \rightarrow-\mathrm{i} t$ and $t_{C} \rightarrow-\mathrm{i} t$. In the former case, one obtains the geometry of global $\mathrm{AdS}_{3}$ with compact imaginary time corresponding to thermal AdS. The latter choice leads instead to the BTZ black hole. Note that the coordinates $t_{N C}$ and $t_{C}$ are related by a simple modular transformation of the boundary torus, $\tau \rightarrow-\frac{1}{\tau}$. Hence if we obtain the partition function for one such solution, it is immediate to obtain the partition function for the other one.

For a generic asymptotically $\mathrm{AdS}_{3}$ solution, the conserved quantities are the energy $H$ and the angular momentum $J$, which corresponds to the momentum in the CFT at the boundary. The partition function then reads:

$$
Z(\beta, \mu)=e^{-S_{E}}=\operatorname{Tr} e^{-\beta H-\mu J}=\operatorname{Tr} e^{2 \pi \mathrm{i} \tau h_{L}} e^{-2 \pi \mathrm{i} \bar{\tau} h_{R}},
$$

where the following definitions have been used for the modular parameter of the torus, $\tau$,

\footnotetext{
${ }^{5} \mathrm{~A}$ well-known example of such a compactification is the case when $X=K 3 \times T^{2}$. An $M 5$-brane on such a manifold is dual to heterotic string theory, which has both a susy and a non-susy sector. Our results, as we shall see, are sensitive to all the excitations independent of their supersymmetries.
} 
and the zero modes of the Virasoro algebra:

$$
\begin{aligned}
\tau & =\mathrm{i} \frac{\beta-\mu}{2 \pi}, & \bar{\tau} & =-\mathrm{i} \frac{\beta+\mu}{2 \pi}, \\
h_{L} & =L_{0}-\frac{c_{L}}{24}=\frac{H-J}{2}, & h_{R} & =\tilde{L}_{0}-\frac{c_{R}}{24}=\frac{H+J}{2} .
\end{aligned}
$$

Note that in Euclidean signature the modular parameter $\tau$ becomes complex, and $\mu$ is purely imaginary. Furthermore, from the knowledge of the partition function, the zero modes of Virasoro generators can be simply obtained as:

$$
h_{L}=\frac{\mathrm{i}}{2 \pi} \frac{\partial S_{E}}{\partial \tau}, \quad h_{R}=\frac{-\mathrm{i}}{2 \pi} \frac{\partial S_{E}}{\partial \bar{\tau}} .
$$

From these generic considerations it is quite easy to obtain the thermal partition function for $\mathrm{AdS}_{3}$. Since global $\mathrm{AdS}_{3}$ corresponds to the NS-NS vacuum for which $L_{0}=\tilde{L}_{0}=0$, we get:

$$
S_{E}=\frac{\mathrm{i} \pi}{12}\left(c_{L} \tau-c_{R} \bar{\tau}\right)
$$

This is the result for the local part of the partition functions, where non-local contributions given by excitations of massless particles can be neglected in the low temperature (large $\beta$ ) limit. By using the modular transformation $\tau \rightarrow-\frac{1}{\tau}$, one gets the partition function for BTZ black holes:

$$
S_{\mathrm{E}, \mathrm{BTZ}}(\tau, \bar{\tau})=S_{E}\left(-\frac{1}{\tau},-\frac{1}{\bar{\tau}}\right)=-\frac{\mathrm{i} \pi}{12}\left(\frac{c_{L}}{\tau}-\frac{c_{R}}{\bar{\tau}}\right)
$$

and, from (3.3), $h_{L}=-\frac{c_{L}}{24 \tau^{2}}$ and $h_{R}=-\frac{c_{R}}{24 \bar{\tau}^{2}}$. Note now that the Helmholtz free energy corresponds to the Euclidean action, $S_{E}=\beta H+\mu J-\mathcal{S}$, so it is easy to get the Cardy formula:

$$
\mathcal{S}_{\mathrm{BTZ}}=2 \pi\left(\sqrt{\frac{c_{L} h_{L}}{6}}+\sqrt{\frac{c_{R} h_{R}}{6}}\right)
$$

which is the expected result and can be obtained without relying on AdS/CFT correspondence. Since the entropy depends on $c_{L, R}$ (with $h_{L, R}$ fixed by (3.2)), we would like to compute the central charges directly in gravity description which, in our context, includes higher derivative corrections. This can be done by exploiting the anomalies of the system; the gravity side suffers from anomalies arising from Chern-Simons couplings, and so does the CFT theory. The important observation here is that the conformal anomalies are proportional to the central charges. It is important to point out that we are not aiming at canceling the anomalies of either theory. We want to obtain the anomalies on both the gravity and the CFT side, and by comparison, a formula for the central charges of the CFT in terms of the charges and coefficients characterizing the theory of gravity, including the higher derivative sector. There are many different but equivalent approaches to this problem $[4,5,10,16]$. Here we outline the line of reasoning and the main results, referring to the previous references for a detailed discussion.

The five dimensional $\mathrm{AdS}_{3} \times \mathrm{S}^{2}$ supergravity background, which is of interest to us, arises as a near-horizon limit of extremal BTZ black holes. The theory arises by reducing 
M-theory on a Calabi-Yau three-fold $X$. From the perspective of M-theory, we wrap $Q_{5}$ M5-branes with unity charge (or equivalently one M5-brane, with charge $Q_{5}$ ) over the four-cycles of a Calabi-Yau three-fold $X$, hence reducing the six-dimensional world-volume of the M5-branes to a $1+1$ dimensional world-sheet spanned by a string in 5 -dimensions. At low energies, this string is described by a chiral $(4,0)$ supersymmetric $\mathrm{CFT}_{2}$. The anomalous gravitational Chern-Simons term in the five dimensional theory arises from the dimensional reduction of the M-theory coupling between the three-form potential $C_{3}$ and an eight-form term, proportional to the Riemann curvature to the fourth power. Each M5-brane is magnetically charged under the three-form potential with unity charge. Now if we call $\left\{\theta^{A}\right\},{ }^{6}$ an integral basis for the cohomolgy group $H^{2}(X, \mathbb{Z})$ of the Calabi-Yau three-fold, and $\left\{\sigma_{A}\right\}$ the dual basis of the homology group $H_{4}(X, \mathbb{Z})$, then the three-form potential reduces to a set of $\mathrm{U}(1)$ connection as $C_{3}=A_{1}^{A} \wedge \theta_{A}$. In the following, we will indicate by $A_{1}$ the linear combination of abelian gauge fields obtained from the element of $H^{2}$ dual to a smooth cycle $P_{0}=P_{0}^{A} \sigma_{A}$ of $H_{4}$, i.e.

$$
A_{1}=a_{A} A_{1}^{A} \quad a_{A} \cdot P_{0}^{B}=\delta_{A}^{B} .
$$

By wrapping the $Q_{5}$ five-branes on the smooth cycles $P_{0}$, one obtains a string in fivedimensional space, charged under the $\mathrm{U}(1)$ connections with charges $P_{0}^{A}$. In our case we are wrapping $p^{A}$ unity charge M5-branes over the $A$-th four-cycle of $\mathrm{CY}_{3}$.

We will come back shortly to the microscopic stringy picture, but first, it is instructive to understand the supergravity picture. We are considering a smooth near-horizon configuration $\mathrm{AdS}_{3} \times \mathrm{S}^{2} \times \mathrm{X}$ of a higher derivative theory including the anomalous Chern-Simons couplings

$$
S_{\text {anml }}=\frac{1}{48} c_{2} \cdot P_{0} \int_{M_{5}} A_{1} \wedge p_{1}
$$

where $p_{1}=-\frac{1}{2(2 \pi)^{2}} \operatorname{Tr}(R \wedge R)$ is the first Pontryagin class of the 2 -form curvature $R$. Note that in this low-energy limit, the branes are interpreted as fluxes. Specifically, the magnetic charges of this solution are given by:

$$
P_{0}^{A}=-\frac{1}{2 \pi} \int_{S^{2}} F^{A}, \quad Q_{5}=-\frac{1}{2 \pi} \int_{S^{2}} F_{1},
$$

where $F^{A}=d A^{A}$ and $F_{1}=d A_{1}$ and we are assuming that the field strength has components only on the two-sphere. These are the same charges of the string configuration. Now the coupling (3.7) suffers from two anomalies connected to diffeomorphism transformations on the tangent bundle and the normal bundle. Practically speaking, these anomalies correspond respectively to the anomalous transformations mapping the boundary onto the boundary and acting on the vectors normal to the boundary. In the stringy perspective, the former corresponds to the tangent bundle anomaly on the string world-sheet, and is connected to the gravitational anomalies of the $\mathrm{CFT}_{2}$. Specifically, if one uses Christoffel connections to define the curvature $R$, then the anomaly in the gravity side corresponds to the non-conservation of the stress-energy tensor. If instead, the spin connection is used,

\footnotetext{
${ }^{6}$ The index A runs from 1 to $b^{2}=b_{4}$, where $b$ is the Betty number of the three-fold $\mathrm{X}$.
} 
then the anomaly corresponds to the antisymmetric part of the stress-energy tensor. The normal bundle anomaly is interpreted as anomalous Lorentz transformation acting on the directions normal to the string world-sheet. Since we are in five dimensions, this group is $\mathrm{SO}(3)$, and is associated to the R-symmetry group $\mathrm{SU}(2)_{L}$ acting on the left-mover degrees of freedom of the $(4,0)$ CFT.

Let us start analyzing the tangent bundle anomaly. On the supergravity side, under an infinitesimal diffeomorphism transformation of the form $x^{\mu} \rightarrow x^{\mu}-\xi^{\mu},{ }^{7}$ the transformations rules are as follows (see $[18,19]$ for a pedagogical discussion):

$$
\delta_{\xi} g_{\mu \nu}=2 \xi_{(\mu \nu)}, \quad \delta_{\xi} \Gamma=\mathrm{d} \xi+[\Gamma, \xi], \quad \delta_{\xi} R=[R, \xi],
$$

where $\xi_{\mu \nu}=\frac{\partial \xi_{\mu}}{\partial x^{\nu}}$. Now the anomalous term (3.7) can be written as:

$$
S_{\text {anml }}=-\frac{1}{(2 \pi)^{2}} \frac{1}{96} c_{2} \cdot P_{0} \int_{M_{5}} A \wedge \operatorname{Tr}(R \wedge R)=\frac{1}{(2 \pi)^{2}} \frac{1}{96} c_{2} \cdot P_{0} \int_{M_{5}} F_{1} \wedge \operatorname{Tr}\left(\Omega_{3}\right),
$$

where, we defined $\Omega_{3}=\operatorname{Tr}\left(\Gamma \mathrm{d} \Gamma+\frac{2}{3} \Gamma^{3}\right)$. The above equality represents the first step of the descent procedure, i.e. $\operatorname{Tr}(R \wedge R)=\mathrm{d} \Omega_{3}$; the integrand proportional to the gauge field strength can be made well-defined by partially integrating it, as the linear combination of gauge fields $A_{1}$ is ill-defined in the presence of magnetic charges. Next, we exploit the fact that the field strength $F_{1}$ has components only over the sphere, to work out the integral and get:

$$
S_{\text {anml }}=-\frac{1}{(2 \pi)} \frac{1}{96} c_{2} \cdot p \int_{A d S_{3}} \operatorname{Tr}\left(\Omega_{3}\right) .
$$

Now, we just need to apply the second step of the descent procedure, i.e. we consider a symmetry variation and write it in terms of an exact form. Since $\delta_{\xi} \Omega_{3}=\mathrm{d} \operatorname{Tr}(v \mathrm{~d} \Gamma)$, we have:

$$
\delta_{\xi} S_{a n m l}=-\frac{1}{(2 \pi)} \frac{1}{96} c_{2} \cdot p \int_{\partial A d S_{3}} \operatorname{Tr}(v \mathrm{~d} \Gamma) .
$$

This shows that the diffeomorphism invariance of the bulk theory is preserved while the invariance of the boundary theory is anomalous. But this anomaly can be equaled to the gravitational anomaly of the $\mathrm{CFT}_{2}$, which can also be computed through the descent procedure, from the four form $I_{4}=-\frac{\left(c_{L}-c_{R}\right) p_{1}}{24}$. We finally have:

$$
\delta_{\xi} S_{C F T}=\frac{c_{R}-c_{L}}{48(2 \pi)} \int_{\partial A d S_{3}} \operatorname{Tr}(v d \Gamma) .
$$

By simple comparison we find the identity:

$$
c_{L}-c_{R}=\frac{1}{2} c_{2} \cdot p .
$$

This is not enough to constrain both the central charges, but we do have another anomaly to take into account, the normal bundle anomaly, which corresponds to R-symmetry anomaly in the $(4,0)$ CFT side. In fact, since our theory is chiral, it will be anomalous only in

\footnotetext{
${ }^{7}$ Note that diffeomorphism anomalies are possible only in even dimensions, so in this context, these anomalies arise as boundary symmetries that are broken in the quantum theory.
} 
the supersymmetric sector (leftmovers in our conventions). The analysis for the normal bundle anomaly turns out to be more involved than its tangent bundle counterpart. We note here that, since the gravity theory we are considering is supersymmetric, the two anomalies must necessarily fit in the same multiplet, i.e. be connected by supersymmetry transformations. Furthermore, as was pointed out in [6] the Chern-Simons term treats the left and right central charges oppositely (we refer also to [20], where a crucial result for the analysis of [6] is found). Here we follow the reasoning of [4]; as we said before, the normal bundle anomaly for a string in 5 dimensions is connected to the subgroup $\mathrm{SO}(3)$ of the Lorentz group, which leaves the string invariant (hence acting on its transverse directions). Specifically, the left-movers superalgebra is an $\mathrm{SO}(3)$ Kac-Moody algebra with level $k$, which is connected, by the AdS/CFT correspondence, to the coefficients of the Chern-Simons terms. Furthermore, the superconformal algebra constrains the (left, in this case) central charge to be equal to $c_{L}=6 k$. All that is left is for us to compute the level $k$. We refrain from giving the details of the calculation; the interested reader should carefully read [4]. The final result, which also includes the contribution from the gauge Chern-Simons coupling $A \wedge F \wedge F$, reads:

$$
k=D_{0}+\frac{1}{12} c_{2} \cdot p,
$$

where $D_{0}=\frac{1}{6} D_{A B C} p^{A} p^{B} p^{C}$ and $D_{A B C}$ are the intersection numbers of $X$. Using (3.14) and (3.15) we finally find the values of the central charges

$$
c_{L}=D_{A B C} p^{A} p^{B} p^{C}+\frac{1}{2} c_{2} \cdot p, \quad c_{R}=D_{A B C} p^{A} p^{B} p^{C}+c_{2} \cdot p .
$$

The result (3.16) depends only on the Chern-Simons terms of the five (or three) dimensional theory, which are anomalous and contribute to both the left (BPS) and the right (non-BPS) central charges of the boundary CFT theory. Hence, since no other anomalous terms should exists in 5D, the above results should be correct at any order in the effective pertubative expansion of the theory.

Now, to obtain an explicit result for the entropy, it is just a matter of finding the value of $h_{L, R}$ for the classes of (non-)BPS $\mathrm{AdS}_{3} \times \mathrm{S}^{2}$ background.

\section{$3.25 \mathrm{D}$ vs $4 \mathrm{D}$ theories and solutions}

We are interested in computing the subleading corrections to the entropy of fourdimensional black hole solutions in $\mathcal{N}=2$ supergravity, with the near-horizon geometry $\mathrm{AdS}_{2} \times \mathrm{S}^{2}$. Hence we need to justify the connection between the results obtained for five dimensional black holes in the previous section and the results we aim to obtain. First of all, as already emphasized, the statistical entropy computation of the five dimensional black holes is sensitive only to the anomalous terms, which do not exist in the four dimensional theory. Secondly, the BTZ background analyzed is obtained as the compatification of an M-theory solution over a Calabi-Yau three-fold and hence as the (non-)BPS solution of a supersymmetric theory. On the other hand, the macroscopic methods available to compute the entropy of supergravity black holes are based on the knowledge of the full 
Lagrangian, and no supersymmetry is required. If we wish to exploit the five-dimensional entropy results for the four-dimensional backgrounds of interest in this work, we need to find the connection between the two theories and the two corresponding backgrounds. The latter is quite obvious; the five dimensional $\mathrm{AdS}_{3} \times \mathrm{S}^{2}$ background is related to the four dimensional $\mathrm{AdS}_{2} \times \mathrm{S}^{2}$ background via a simple circle reduction. Analogously, the five dimensional supergravity theory, with Weyl squared higher-derivative corrections [15] can be reduced over a circle and the reduction procedure has been worked out in great detail in [14]. The results showed the presence of some new higher-derivative terms, belonging to no known four derivatives invariant in four dimensions. In retrospect, it is very easy to guess that the five-dimensional gravitational Chern-Simons term $A_{5} \wedge R_{5} \wedge R_{5}$, with $R_{5}$ being the Riemann tensor in $5 \mathrm{D}$, will reduce to terms proportional to $X_{4}^{0} R_{4} \wedge R_{4}$ in $4 \mathrm{D}$, which is squared in the curvatures, but not proportional to the squared Weyl tensor.

Shortly after, these curvature squared terms were found in the purely four-dimensional context, as part of the bosonic sector of a new class of higher derivative invariants, TLog invariants, built out of a non-linear chiral multiplet [1]. Contrary to the Weyl squared invariant, which depends only on superconformal gravity fields, the new class of invariants depends explicitly on matter (or gauge) multiplets. Furthermore, by combining the TLog invariant, for a constant compensating multiplet configuration, and the Weyl squared Lagrangian, one obtains the Gauss-Bonnet density, which is a topological invariant of four dimensional gravity theory.

We want to stress that it is of utmost importance for the four-dimensional theory under consideration to be the exact reduction of the supersymmetrization of the five-dimensional gravitational Chern-Simons terms. Even though the entropy computation depends only on the two Chern-Simons terms, supersymmetry of the full action plays a crucial role. This is the result presented in (2.6). We refer the reader to the last section of [9] for further details.

At this point, it should be clear that the results obtained in the previous section can also be used in the four-dimensional context without any modification. This would require only the identification of the reduced background and the reduced theories correctly $[9,14]$. We are now ready to present the details of the entropy computation for a class of BPS and non-BPS black hole solutions, and show the exact agreement with the entropy results (3.6) and (3.16).

\subsection{Entropy of (non-)BPS black holes}

The classes of black holes of interest to this work are obtained as solutions of superconformal gravity coupled to three vector multiplets, labelled by the indices A, B and C. However the number of vector multiplets in our solution is by no means constrained, i.e. the results we obtain in this section can be generalized to any number of vector multiplets. Doing so would slightly change the physical interpretation, as then we would be considering an effective low energy description of M-theory compactified over a Calabi-Yau manifold [10] (see also [8]).

We start with BPS black hole solutions, which were first analyzed in $[11,12]$. On imposing full supersymmetry of the background, sufficient conditions extremizing the entropy 
function can be found. They are:

$$
v_{1}=v_{2}=\frac{16}{z \bar{z}}, \quad \mathcal{F}_{01}^{-I}=e^{I}-\mathrm{i} \frac{v_{1}}{v_{2}} p^{I}-\frac{1}{2} \bar{X}^{I} v_{1} z=0, \quad \frac{\bar{F}_{I}}{\bar{z}}-\frac{F_{I}}{z}=-\frac{\mathrm{i}}{4} q_{I} .
$$

The first two conditions are sufficient to make the whole TLog invariant collapse to zero [9]. The only non-vanishing terms in the higher derivative sector come from the highest component of the Weyl squared invariant. The conditions above are steadily solved by:

$$
e^{I}=4\left(\frac{\bar{X}^{I}}{\bar{z}}+\frac{X^{I}}{z}\right), \quad p^{I}=4 \mathrm{i}\left(\frac{\bar{X}^{I}}{\bar{z}}-\frac{X^{I}}{z}\right)
$$

from which we derive a general formula for the entropy, given by:

$$
S_{\mathrm{BH}}=2 \pi\left[-\frac{1}{2} e^{I} q_{I}-16 \mathrm{i}\left(\frac{F}{z^{2}}-\frac{\bar{F}}{\bar{z}^{2}}\right)\right] .
$$

Note that the entropy still depends on the electric fields $e^{I}$, the vector multiplet scalars $X^{I}$ and the T-tensor component $z$. To fix those we exploit the invariance of the equations of motion, derived from the Lagrangian (2.3) with the prepotential $(2.1)$, under $\mathrm{SO}(2,2)=$ $\mathrm{SL}(2, \mathbb{Z}) \times \mathrm{SL}(2, \mathbb{Z})$ T-duality. This means that we can choose a representative set of charges and electric fields, to simplify the computation. In this case, we can choose as representative satisfying the $\mathbb{Z}_{2}$ symmetry that the four dimensional theory inherits from M-theory, i.e.:

$$
p^{i} \rightarrow p^{A}, \quad p^{0} \rightarrow-p^{0}, \quad e^{A} \rightarrow-e^{A}, \quad e^{0} \rightarrow e^{0}, \quad X^{A} \rightarrow-\bar{X}^{A}, \quad X^{0} \rightarrow \bar{X}^{0}, \quad z \rightarrow \bar{z} .
$$

Thus we can choose a gauge (or exploit $\mathbb{Z}_{2}$ invariance) to fix the charges and the moduli as:

$$
p^{0}=0, \quad e^{A}=0, \quad X^{A}=\mathrm{i} y^{A}, \quad X^{0}=y^{0},
$$

with $z, y^{i} \in \mathbb{R}$. Note that this also implies $q^{A}=0$.

For the higher derivative theory (2.3), the supersymmetric attractors conditions (3.17) are satisfied by:

$$
\begin{aligned}
& w=1, \quad v_{1}=v_{2}=16, \quad x^{A}=\frac{1}{8} \mathrm{i} p^{A}, \quad e^{A}=0, \\
& x^{0}=\frac{1}{8} \sqrt{\frac{d_{A B C} p^{A} p^{B} p^{C}+256 d_{A} p^{A}}{-q_{0}}}, \quad e^{0}=\sqrt{\frac{d_{A B C} p^{A} p^{B} p^{C}+256 d_{A} p^{A}}{-q_{0}}},
\end{aligned}
$$

for $q_{0}<0$ and $d_{A B C} p^{A} p^{B} p^{C}+256 d_{A} p^{A}>0$. Note at this point that the KK-charge $q_{0}$ in four dimensions is identified with the angular momentum $J$ of the BTZ $\times S^{2}$ solution in $5 \mathrm{D}$, for which, in the extremal limit, $H=|J|$. The class of BPS solutions analyzed satisfies $H=-J$, so from eq. (3.2) we get $h_{L}=0$ and $h_{R}=-J=-q_{0}>0$. This indeed leads to the result of [11], i.e.,

$$
S_{\mathrm{BH}}=2 \pi \sqrt{-q_{0}\left(d_{A B C} p^{A} p^{B} p^{C}+256 d_{A} p^{A}\right)} .
$$


We point out that the constraints imposed by the BPS attractors are very stringent and allow for an analytical solution for all the fields, even when the higher derivative sector is considered. That is why the final result is already in the form expected from the analogous statistical computation, i.e. the Cardy formula. As we stressed before, the situation will not be quite as simple for the non-BPS solutions, as a closed form analytical solution will, in general, be missing.

Next, we procede with the computation of the entropy of non-BPS solutions, studied in $[21,22]$. This class of solutions exists for the two derivative theory, $d_{A}=0$, and has the same charge configuration as the BPS solution above except for one difference, residing in a simple sign change for the electric field $e^{0}$, consistent with the $\mathbb{Z}_{2}$ duality symmetry of the two derivative action. The auxiliary field $z$ cannot be fixed by this procedure and we need to solve for it via the extremization equations. The results hence read:

$$
\begin{aligned}
w & =\frac{1}{2}, \quad v_{1}=v_{2}=16, & x^{A} & =\frac{1}{8} \mathrm{i} p^{A}, \quad e^{A}=0, \\
x^{0} & =\frac{1}{8} \sqrt{\frac{d_{A B C} p^{A} p^{B} p^{C}}{q_{0}}}, & e^{0} & =-\sqrt{\frac{d_{A B C} p^{A} p^{B} p^{C}}{q_{0}}},
\end{aligned}
$$

for $q_{0}>0$ and $d_{A B C} p^{A} p^{B} p^{C}>0$. The leading order entropy reads:

$$
S_{\mathrm{BH}}=2 \pi \sqrt{q_{0}\left(d_{A B C} p^{A} p^{B} p^{C}\right)} .
$$

To compute the first order corrections in $d_{A}$ we just need to plug this leading order solution into the full entropy function, as explained before. Note that, if we would have used Wald formalism, we would have needed the full near-horizon solution for the parameters, so we could not have used the solution presented here, which was found from an $\mathcal{N}=2$ two derivative theory [22]. Note again that, since we are flipping the sign of $q_{0}=J$, the condition of extremality $H=J$ forces $h_{R}=0$ and $h_{L}=J=q_{0}>0$. So the first subleading order correction to the entropy of this class of black holes is now given by:

$$
S_{\mathrm{BH}}=2 \pi \sqrt{q_{0}\left(d_{A B C} p^{A} p^{B} p^{C}\right)}\left(1+\frac{64 d_{A} p^{A}}{d_{A B C} p^{A} p^{B} p^{C}}\right),
$$

which corresponds, as we announced before, to the correct expansion up to the first order of Cardy formula (eq. (3.6)). Hence we have resolved the puzzle concerning the macroscopic entropy of non-BPS black holes in 5D/4D.

\section{Results and conclusions}

In this work we have resolved an entropy puzzle for four dimensional non-BPS black holes of $\mathcal{N}=2$ supergravity. We find perfect agreement, in the thermodynamic limit of large charges, between our macroscopic entropy results and the expected statistical entropy computed in [5], even for non-supersymmetric black holes. The key to obtain this result is the knowledge of the exact connection between the five and four-dimensional theories. Specifically, the five-dimensional result is obtained from the anomaly calculations in $5 \mathrm{D}$, where 
the anomalous terms contributing to the entropy are the Chern-Simons interactions. To match this result in $4 \mathrm{D}$, the need for the full reduction of the supersymmetric gravitational Chern-Simons term was imperative [14], and the relevant results in [1, 9] have been used extensively in this work. Based on the results of [4-6], it would surely seem that no other higher order invariant would contribute to the entropy of (non-)BPS black holes in four dimensions, simply because the statistical $5 \mathrm{D}$ results that hinge on anomalies, cannot be corrected further, as no more anomalous terms are supposed to play a role at any order in the perturbative expansion of the 5D theory. There might exist, however, other $\mathcal{N}=2$ higher derivative invariants in $4 \mathrm{D}$ that contribute non-trivially to the entropy of the class of black hole analyzed: this would signal the presence in the 5D theory of new, so far unknown and unexpected, anomalous higher derivative interaction term(s), or equivalently, the breaking of the validity of the Cardy formula. ${ }^{8}$ In the context analyzed in this paper, however, the microscopic counting and the macroscopic degeneracies are exactly linked by the Cardy formula as we explained at length in section 3.1 (see also [5, 23]). The computation is solid and little doubt exists about its validity. In fact, even though in the previous sections we restricted ourselves to first order corrections to the entropy, ${ }^{9}$ one can push the computation forward, by iteratively computing the higher order corrections to the near-horizon parameter and entropy. It turns out that the series expansion of entropy coincides exactly with the series expansion of the square root, i.e.

$$
\begin{aligned}
S_{\mathrm{BH}}= & 2 \pi \sqrt{q_{0}\left(d_{A B C} p^{A} p^{B} p^{C}\right)}\left(1+64 u-2048 u^{2}+131072 u^{3}-10485760 u^{4}\right. \\
& \left.+939524096 u^{5}+\ldots\right) \\
= & 2 \pi \sqrt{q_{0}\left(d_{A B C} p^{A} p^{B} p^{C}+128 d_{A} p^{A}\right)}
\end{aligned}
$$

with $u=\frac{d_{A} p^{A}}{d_{A B C} p^{A} p^{B} p^{C}}$ being the smallness parameter. Hence, as consistency dictates, the statistical entropy computed from anomalies matches exactly the macroscopic entropy obtained from the knowledge of the full effective action containing the anomalous terms. Of course, this proves that, for the class on large black holes considered herein, the anomaly result is reliable also in the non-supersymmetric sector. Nevertheless, there exist indications that the anomaly results might not work for every class of black holes. In fact, although the supersymmetric gravitational Chern-Simons term is the most analyzed higher derivative invariant in 5D, other invariants were recently constructed [24]. These invariants also include the Gauss-Bonnet density in 5D, which was analyzed in the context of black hole entropy computations many years before [25]. As it turns out [26], small black holes seem to escape the description in terms of the Chern-Simons terms, and instead receive corrections from the disconnected Gauss-Bonnet sector. This means that there might be some hidden caveat in the reasoning of [5] which is evidently contradicted in the presence of small black holes. If the $5 \mathrm{D}$ theory possesses many sectors, each of which is connected to special classes

\footnotetext{
${ }^{8}$ For instance, in [23], the proper matching between the microscopic and macroscopic entropy is obtained through the use of indices, while the Cardy formula fails to capture important subleties.

${ }^{9}$ This is, in principle, the maximal accuracy we can reach by considering the lowest order higher derivative action.
} 
of black holes, then it is obvious to assume that something similar would also happen in $4 \mathrm{D}$ theory, i.e. other higher derivative invariants, of the first order in the perturbative expansion in $d_{A}$ might exist in $\mathcal{N}=2$ theory. Furthermore, it would be important to understand how exactly the anomaly procedure that led to the correct entropy results for the classes of black holes analyzed, fails to capture information about different classes, and its exact range of applicability. We plan on addressing these issues in the near-future.

\section{Acknowledgments}

We are thankful to Daniel Butter, Suvankar Dutta, Sameer Murthy, Bernard de Wit, Dileep Jatkar, Bindusar Sahoo and Ashoke Sen for discussions at various stages of this work. Ivano Lodato thanks the organizers of NSM Mohali, where this work was first presented. NB and IL are partly supported by a DST Ramanujan Grant. Finally, we are thankful to the people of India for their generous support to basic science.

\section{A Conventions and useful identities}

We use the Pauli-Källén convention. Spacetime indices are denoted $\mu, \nu, \ldots$, Lorentz indices are denoted $a, b, \ldots$, and $\mathrm{SU}(2)$ indices are denoted $i, j, \ldots$. The Lorentz metric is $\eta_{a b}=$ $\operatorname{diag}(-1,1,1,1)$ and the anti-symmetric tensor $\varepsilon_{a b c d}$ is imaginary, with $\varepsilon_{0123}=-\mathrm{i}=-\varepsilon^{0123}$.

An anti-symmetric two-form $F_{a b}$ satisfies the following identities:

$$
F_{a b}^{ \pm}=\frac{1}{2}\left(F_{a b} \pm \tilde{F}_{a b}\right), \quad \tilde{F}_{a b}=\frac{1}{2} \varepsilon_{a b c d} F^{c d}, \quad \tilde{F}_{a b}^{ \pm}= \pm F_{a b}^{ \pm} .
$$

We always apply symmetrization and anti-symmetrization with unit strength, so that $F_{[a b]}=F_{a b}$ and $F_{(\alpha \beta)}=F_{\alpha \beta}$. Furthermore, the following useful identities for products of (anti-)selfdual tensors are noted,

$$
\begin{aligned}
G_{[a[c}^{ \pm} H_{d] b]}^{ \pm} & = \pm \frac{1}{8} G_{e f}^{ \pm} H^{ \pm e f} \varepsilon_{a b c d}-\frac{1}{4}\left(G_{a b}^{ \pm} H_{c d}^{ \pm}+G_{c d}^{ \pm} H_{a b}^{ \pm}\right), \\
G_{a b}^{ \pm} H^{\mp c d}+G^{ \pm c d} H_{a b}^{\mp} & =4 \delta_{[a}^{[c} G_{b] e}^{ \pm} H^{\mp d] e}, \\
\frac{1}{2} \varepsilon^{a b c d} G_{[c}^{ \pm e} H_{d] e}^{ \pm} & = \pm G^{ \pm[a}{ }_{e} H^{ \pm b] e} \\
G^{ \pm a c} H_{c}^{ \pm b}+G^{ \pm b c} H_{c}^{ \pm a} & =-\frac{1}{2} \eta^{a b} G^{ \pm c d} H_{c d}^{ \pm}, \\
G^{ \pm a c} H_{c}^{\mp b} & =G^{ \pm b c} H_{c}^{\mp a}, \\
G^{ \pm a b} H_{a b}^{\mp} & =0 .
\end{aligned}
$$

Finally, we remind the reader that $\mathrm{SU}(2)$ indices are swapped by complex conjugation, $\left(T_{a b i j}\right)^{*}=T_{a b}{ }^{i j}$, and we make use of the invariant $\mathrm{SU}(2)$ tensor $\varepsilon^{i j}$ and $\varepsilon_{i j}$ defined as $\varepsilon^{\underline{12}}=\varepsilon_{\underline{12}}=1$ with $\varepsilon^{i j} \varepsilon_{k j}=\delta_{k}^{i}$. 


\begin{tabular}{|c|cccccccc|ccc|cc|}
\hline & \multicolumn{10}{|c|}{ Weyl Multiplet Components } & Parameters \\
\hline field & $e_{\mu}{ }^{a}$ & $\psi_{\mu}{ }^{i}$ & $b_{\mu}$ & $A_{\mu}$ & $\mathcal{V}_{\mu}{ }^{i} j_{j}$ & $T_{a b}{ }^{i j}$ & $\chi^{i}$ & $D$ & $\omega_{\mu}^{a b}$ & $f_{\mu}{ }^{a}$ & $\phi_{\mu}{ }^{i}$ & $\epsilon^{i}$ & $\eta^{i}$ \\
\hline$w$ & -1 & $-\frac{1}{2}$ & 0 & 0 & 0 & 1 & $\frac{3}{2}$ & 2 & 0 & 1 & $\frac{1}{2}$ & $-\frac{1}{2}$ & $\frac{1}{2}$ \\
\hline$c$ & 0 & $-\frac{1}{2}$ & 0 & 0 & 0 & -1 & $-\frac{1}{2}$ & 0 & 0 & 0 & $-\frac{1}{2}$ & $-\frac{1}{2}$ & $-\frac{1}{2}$ \\
\hline$\gamma_{5}$ & & + & 1 & & & + & & & & - & + & - \\
\hline
\end{tabular}

Table 1. Weyl and chiral weights $\left(w\right.$ and $c$ ) and fermion chirality $\left(\gamma_{5}\right)$ of the Weyl multiplet component fields and the supersymmetry transformation parameters.

\section{B Superconformal mutiplets - (covariant) Weyl, chiral, vector multi- plets}

Recall that the superconformal algebra comprises the generators of the general-coordinate, local Lorentz, dilatation, special conformal, chiral SU(2) and U(1), supersymmetry (Q) and special supersymmetry $(\mathrm{S})$ transformations. The gauge fields associated with generalcoordinate transformations $\left(e_{\mu}{ }^{a}\right)$, dilatations $\left(b_{\mu}\right)$, R-symmetry $\left(\mathcal{V}_{\mu}{ }_{j}{ }_{j}\right.$ and $\left.A_{\mu}\right)$ and Qsupersymmetry $\left(\psi_{\mu}^{i}\right)$ are independent fields. The remaining gauge fields associated with the Lorentz $\left(\omega_{\mu}^{a b}\right)$, special conformal $\left(f_{\mu}{ }^{a}\right)$ and S-supersymmetry transformations $\left(\phi_{\mu}{ }^{i}\right)$ are composite objects. The multiplet also contains three other fields: a Majorana spinor doublet $\chi^{i}$, a scalar $D$, and a selfdual Lorentz tensor $T_{a b i j}$, which is anti-symmetric in $[a b]$ and $[i j]$. The Weyl and chiral weights have been collected in table 1. Under Q-supersymmetry, S-supersymmetry and special conformal transformations, the Weyl multiplet fields transform as

$$
\begin{aligned}
\delta e_{\mu}{ }^{a} & =\bar{\epsilon}^{i} \gamma^{a} \psi_{\mu i}+\bar{\epsilon}_{i} \gamma^{a} \psi_{\mu}{ }^{i} \\
\delta \psi_{\mu}{ }^{i} & =2 \mathcal{D}_{\mu} \epsilon^{i}-\frac{1}{8} T_{a b}{ }^{i j} \gamma^{a b} \gamma_{\mu} \epsilon_{j}-\gamma_{\mu} \eta^{i} \\
\delta b_{\mu} & =\frac{1}{2} \bar{\epsilon}^{i} \phi_{\mu i}-\frac{3}{4} \bar{\epsilon}^{i} \gamma_{\mu} \chi_{i}-\frac{1}{2} \bar{\eta}^{i} \psi_{\mu i}+\text { h.c. }+\Lambda_{\mathrm{K}}^{a} e_{\mu a}, \\
\delta A_{\mu} & =\frac{1}{2} \mathrm{i} \bar{\epsilon}^{i} \phi_{\mu i}+\frac{3}{4} \mathrm{i} \bar{\epsilon}^{i} \gamma_{\mu} \chi_{i}+\frac{1}{2} \mathrm{i} \bar{\eta}^{i} \psi_{\mu i}+\text { h.c. }, \\
\delta \mathcal{V}_{\mu}{ }^{i}{ }_{j} & =2 \bar{\epsilon}_{j} \phi_{\mu}{ }^{i}-3 \bar{\epsilon}_{j} \gamma_{\mu} \chi^{i}+2 \bar{\eta}_{j} \psi_{\mu}{ }^{i}-\text { (h.c.; traceless), } \\
\delta T_{a b}{ }^{i j} & =8 \bar{\epsilon}^{[i} R(Q)_{a b}{ }^{j]} \\
\delta \chi^{i} & =-\frac{1}{12} \gamma^{a b} \not D T_{a b}{ }^{i j} \epsilon_{j}+\frac{1}{6} R(\mathcal{V})_{\mu \nu}{ }_{j} \gamma^{\mu \nu} \epsilon^{j}-\frac{1}{3} \mathrm{i} R_{\mu \nu}(A) \gamma^{\mu \nu} \epsilon^{i}+D \epsilon^{i}+\frac{1}{12} \gamma_{a b} T^{a b i j} \eta_{j}, \\
\delta D & =\bar{\epsilon}^{i} \not D \chi_{i}+\bar{\epsilon}_{i} \not D \chi^{i} .
\end{aligned}
$$

Here $\epsilon^{i}$ and $\epsilon_{i}$ denote the spinorial parameters of Q-supersymmetry, $\eta^{i}$ and $\eta_{i}$ those of Ssupersymmetry, and $\Lambda_{\mathrm{K}}{ }^{a}$ is the transformation parameter for special conformal boosts. The full superconformally covariant derivative is denoted by $D_{\mu}$, while $\mathcal{D}_{\mu}$ denotes a covariant derivative with respect to Lorentz, dilatation, chiral $\mathrm{U}(1)$ and $\mathrm{SU}(2)$ transformations,

$$
\mathcal{D}_{\mu} \epsilon^{i}=\left(\partial_{\mu}-\frac{1}{4} \omega_{\mu}^{c d} \gamma_{c d}+\frac{1}{2} b_{\mu}+\frac{1}{2} \mathrm{i} A_{\mu}\right) \epsilon^{i}+\frac{1}{2} \mathcal{V}_{\mu}{ }^{i} \epsilon^{j}
$$


The covariant curvatures are given by

$$
\begin{aligned}
& R(P)_{\mu \nu}^{a}=2 \partial_{[\mu} e_{\nu]}^{a}+2 b_{[\mu} e_{\nu]}^{a}-2 \omega_{[\mu}^{a b} e_{\nu] b}-\frac{1}{2}\left(\bar{\psi}_{[\mu}^{i} \gamma^{a} \psi_{\nu] i}+\text { h.c. }\right), \\
& R(Q)_{\mu \nu}^{i}=2 \mathcal{D}_{[\mu} \psi_{\nu]}^{i}-\gamma_{[\mu} \phi_{\nu]}^{i}-\frac{1}{8} T^{a b i j} \gamma_{a b} \gamma_{[\mu} \psi_{\nu] j}, \\
& R(A)_{\mu \nu}=2 \partial_{[\mu} A_{\nu]}-\mathrm{i}\left(\frac{1}{2} \bar{\psi}_{[\mu}^{i} \phi_{\nu] i}+\frac{3}{4} \bar{\psi}_{[\mu}^{i} \gamma_{\nu]} \chi_{i}-\text { h.c. }\right), \\
& R(\mathcal{V})_{\mu \nu}{ }^{i}{ }_{j}=2 \partial_{[\mu} \mathcal{V}_{\nu]}{ }^{i}{ }_{j}+\mathcal{V}_{[\mu k}{ }^{i}{ }^{\prime} \mathcal{V}_{\nu]}{ }_{j}{ }_{j}+2\left(\bar{\psi}_{[\mu}{ }^{i} \phi_{\nu] j}-\bar{\psi}_{[\mu j} \phi_{\nu]}{ }^{i}\right)-3\left(\bar{\psi}_{[\mu}{ }^{i} \gamma_{\nu]} \chi_{j}-\bar{\psi}_{[\mu j} \gamma_{\nu]} \chi^{i}\right) \\
& -\delta_{j}{ }^{i}\left(\bar{\psi}_{[\mu}{ }^{k} \phi_{\nu] k}-\bar{\psi}_{[\mu k} \phi_{\nu]}{ }^{k}\right)+\frac{3}{2} \delta_{j}{ }^{i}\left(\bar{\psi}_{[\mu}{ }^{k} \gamma_{\nu]} \chi_{k}-\bar{\psi}_{[\mu k} \gamma_{\nu]} \chi^{k}\right), \\
& R(M)_{\mu \nu}^{a b}=2 \partial_{[\mu} \omega_{\nu]}^{a b}-2 \omega_{[\mu}^{a c} \omega_{\nu] c}^{b}-4 f_{[\mu}^{[a} e_{\nu]}^{b]}+\frac{1}{2}\left(\bar{\psi}_{[\mu}^{i} \gamma^{a b} \phi_{\nu] i}+\text { h.c. }\right) \\
& +\left(\frac{1}{4} \bar{\psi}_{\mu}^{i} \psi_{\nu}{ }^{j} T^{a b}{ }_{i j}-\frac{3}{4} \bar{\psi}_{[\mu}{ }^{i} \gamma_{\nu]} \gamma^{a b} \chi_{i}-\bar{\psi}_{[\mu}{ }^{i} \gamma_{\nu]} R(Q)^{a b}{ }_{i}+\text { h.c. }\right) \text {, } \\
& R(D)_{\mu \nu}=2 \partial_{[\mu} b_{\nu]}-2 f_{[\mu}^{a} e_{\nu] a}-\frac{1}{2} \bar{\psi}_{[\mu}^{i} \phi_{\nu] i}+\frac{3}{4} \bar{\psi}_{[\mu}^{i} \gamma_{\nu]} \chi_{i}-\frac{1}{2} \bar{\psi}_{[\mu i} \phi_{\nu]}^{i}+\frac{3}{4} \bar{\psi}_{[\mu i} \gamma_{\nu]} \chi^{i}, \\
& R(S)_{\mu \nu}{ }^{i}=2 \mathcal{D}_{[\mu} \phi_{\nu]}^{i}-2 f_{[\mu}{ }^{a} \gamma_{a} \psi_{\nu]}{ }^{i}-\frac{1}{8} \not D T_{a b}{ }^{i j} \gamma^{a b} \gamma_{[\mu} \psi_{\nu] j}-\frac{3}{2} \gamma_{a} \psi_{[\mu}{ }^{i} \bar{\psi}_{\nu]}{ }^{j} \gamma^{a} \chi_{j} \\
& +\frac{1}{4} R(\mathcal{V})_{a b}{ }^{i}{ }_{j} \gamma^{a b} \gamma_{[\mu} \psi_{\nu]}{ }^{j}+\frac{1}{2} \mathrm{i} R(A)_{a b} \gamma^{a b} \gamma_{[\mu} \psi_{\nu]}{ }^{i}, \\
& R(K)_{\mu \nu}^{a}=2 \mathcal{D}_{[\mu} f_{\nu]}^{a}-\frac{1}{4}\left(\bar{\phi}_{[\mu}^{i} \gamma^{a} \phi_{\nu] i}+\bar{\phi}_{[\mu i} \gamma^{a} \phi_{\nu]}{ }^{i}\right) \\
& +\frac{1}{4}\left(\bar{\psi}_{\mu}^{i} D_{b} T^{b a}{ }_{i j} \psi_{\nu}{ }^{j}-3 e_{[\mu}{ }^{a} \psi_{\nu]}{ }^{i} \not D \chi_{i}+\frac{3}{2} D \bar{\psi}_{[\mu}^{i} \gamma^{a} \psi_{\nu] j}\right. \\
& \left.-4 \bar{\psi}_{[\mu}{ }^{i} \gamma_{\nu]} D_{b} R(Q)^{b a}{ }_{i}+\text { h.c. }\right) \text {. }
\end{aligned}
$$

The connections $\omega_{\mu}^{a b}, \phi_{\mu}{ }^{i}$ and $f_{\mu}^{a}$ are algebraically determined by imposing the conventional constraints

$$
\begin{aligned}
R(P)_{\mu \nu}{ }^{a}=0, \quad \gamma^{\mu} R(Q)_{\mu \nu}{ }^{i}+\frac{3}{2} \gamma_{\nu} \chi^{i} & =0, \\
e^{\nu}{ }_{b} R(M)_{\mu \nu a}{ }^{b}-\mathrm{i} \tilde{R}(A)_{\mu a}+\frac{1}{8} T_{a b i j} T_{\mu}{ }^{b i j}-\frac{3}{2} D e_{\mu a} & =0 .
\end{aligned}
$$

Their solution is given by

$$
\begin{aligned}
\omega_{\mu}{ }^{a b} & =-2 e^{\nu[a} \partial_{[\mu} e_{\nu]}^{b]}-e^{\nu[a} e^{b] \sigma} e_{\mu c} \partial_{\sigma} e_{\nu}^{c}-2 e_{\mu}^{[a} e^{b] \nu} b_{\nu}-\frac{1}{4}\left(2 \bar{\psi}_{\mu}^{i} \gamma^{[a} \psi_{i}^{b]}+\bar{\psi}^{a i} \gamma_{\mu} \psi_{i}^{b}+\text { h.c. }\right), \\
\phi_{\mu}{ }^{i} & =\frac{1}{2}\left(\gamma^{\rho \sigma} \gamma_{\mu}-\frac{1}{3} \gamma_{\mu} \gamma^{\rho \sigma}\right)\left(\mathcal{D}_{\rho} \psi_{\sigma}{ }^{i}-\frac{1}{16} T^{a b i j} \gamma_{a b} \gamma_{\rho} \psi_{\sigma j}+\frac{1}{4} \gamma_{\rho \sigma} \chi^{i}\right), \\
f_{\mu}{ }^{\mu} & =\frac{1}{6} R(\omega, e)-D-\left(\frac{1}{12} e^{-1} \varepsilon^{\mu \nu \rho \sigma} \bar{\psi}_{\mu}{ }^{i} \gamma_{\nu} \mathcal{D}_{\rho} \psi_{\sigma i}-\frac{1}{12} \bar{\psi}_{\mu}{ }^{i} \psi_{\nu}{ }^{j} T^{\mu \nu}{ }_{i j}-\frac{1}{4} \bar{\psi}_{\mu}{ }^{i} \gamma^{\mu} \chi_{i}+\text { h.c. }\right) .
\end{aligned}
$$

We will also need the bosonic part of the expression for the uncontracted connection $f_{\mu}{ }^{a}$,

$$
f_{\mu}^{a}=\frac{1}{2} R(\omega, e)_{\mu}^{a}-\frac{1}{4}\left(D+\frac{1}{3} R(\omega, e)\right) e_{\mu}{ }^{a}-\frac{1}{2} \mathrm{i} \tilde{R}(A)_{\mu}{ }^{a}+\frac{1}{16} T_{\mu b}{ }^{i j} T^{a b}{ }_{i j},
$$

where $R(\omega, e)_{\mu}{ }^{a}=R(\omega)_{\mu \nu}{ }^{a b} e_{b}{ }^{\nu}$ is the non-symmetric Ricci tensor, and $R(\omega, e)$ the corresponding Ricci scalar. The curvature $R(\omega)_{\mu \nu}^{a b}$ is associated with the spin connection field $\omega_{\mu}^{a b}$. 
It is convenient to modify two of the curvatures by including suitable covariant terms,

$$
\begin{aligned}
\mathcal{R}(M)_{a b}{ }^{c d} & =R(M)_{a b}{ }^{c d}+\frac{1}{16}\left(T_{a b i j} T^{c d i j}+T_{a b}{ }^{i j} T^{c d}{ }_{i j}\right), \\
\mathcal{R}(S)_{a b}{ }^{i} & =R(S)_{a b}{ }^{i}+\frac{3}{4} T_{a b}{ }^{i j} \chi_{j},
\end{aligned}
$$

where we observe that $\gamma^{a b}(\mathcal{R}(S)-\mathcal{R}(S))_{a b}{ }^{i}=0$. The modified curvature $\mathcal{R}(M)_{a b}{ }^{c d}$ satisfies the following relations,

$$
\begin{aligned}
\mathcal{R}(M)_{\mu \nu}{ }^{a b} e^{\nu}{ }_{b} & =\mathrm{i} \tilde{R}(A)_{\mu \nu} e^{\nu a}+\frac{3}{2} D e_{\mu}{ }^{a}, \\
\frac{1}{4} \varepsilon_{a b}{ }^{e f} \varepsilon^{c d}{ }_{g h} \mathcal{R}(M)_{e f}{ }^{g h} & =\mathcal{R}(M)_{a b}{ }^{c d}, \\
\varepsilon_{c \text { dea }} \mathcal{R}(M)^{c d e}{ }_{b} & =\varepsilon_{\text {becd }} \mathcal{R}(M)_{a}{ }^{e c d}=2 \tilde{R}(D)_{a b}=2 \mathrm{i} R(A)_{a b} .
\end{aligned}
$$

The first of these relations corresponds to the third constraint given in (B.4), while the remaining equations follow from combining the curvature constraints with the Bianchi identities. Note that the modified curvature does not satisfy the pair exchange property; instead we have,

$$
\mathcal{R}(M)_{a b}{ }^{c d}=\mathcal{R}(M)^{c d}{ }_{a b}+4 i \delta_{[a}^{[c} \tilde{R}(A)_{b]}{ }^{d]} .
$$

Now that the gauge fields of the superconformal agebra have been introduced, the matter multiplets can be discussed. A covariant version of the Weyl multiplet will also be presented. We refer to the original paper [27] for detailed information about the non-linear multiplet.

We start from the chiral multiplet which is typically obtained by imposing the lowest component, the scalar $A$, to be a conformal primary (i.e. S-susy invariant) transforming chirally under Q-susy. The superconformal algebra closes on the following chiral multiplet representation:

$$
\Phi=\left(A, \psi_{i}, B_{i j}, F_{a b}^{-}, \Lambda_{i}, C\right)
$$

where $A$ and $C$ are complex scalars, $\psi_{i}$ and $\Lambda_{i}$ are $\mathrm{SU}(2)$ doublets of chiral fermions, $B_{i j}$ is a complex $\mathrm{SU}(2)$ triplet and $F_{a b}^{-}$is simply an anti-selfdual Lorentz tensor. Each component of the chiral multiplet is characterized by two numbers, $w$ and $c$, called the Weyl and the chiral weight. For chiral multiplets these weights are related by $c=-w$. In that case the Weyl weight of $A$ equals $w$ and the highest- $\theta$ component $C$ has Weyl weight $w+2$ (the list of all the weights assignments is given in table 2). The Q- and S- transformation rules of a generic chiral multiplet read:

$$
\begin{aligned}
\delta A & =\bar{\epsilon}^{i} \Psi_{i}, \\
\delta \Psi_{i} & =2 \not D A \epsilon_{i}+B_{i j} \epsilon^{j}+\frac{1}{2} \gamma^{a b} F_{a b}^{-} \varepsilon_{i j} \epsilon^{j}+2 w A \eta_{i}, \\
\delta B_{i j} & =2 \bar{\epsilon}_{(i} \not D \Psi_{j)}-2 \bar{\epsilon}^{k} \Lambda_{(i} \varepsilon_{j) k}+2(1-w) \bar{\eta}_{(i} \Psi_{j)}, \\
\delta F_{a b}^{-} & =\frac{1}{2} \varepsilon^{i j} \bar{\epsilon}_{i} \not D \gamma_{a b} \Psi_{j}+\frac{1}{2} \bar{\epsilon}^{i} \gamma_{a b} \Lambda_{i}-\frac{1}{2}(1+w) \varepsilon^{i j} \bar{\eta}_{i} \gamma_{a b} \Psi_{j},
\end{aligned}
$$




\begin{tabular}{|c|cccccc|}
\hline & $A$ & $\psi_{i}$ & $B_{i j}$ & $F_{a b}^{-}$ & $\Lambda_{i}$ & $C$ \\
\hline$w$ & $w$ & $w+\frac{1}{2}$ & $w+1$ & $w+1$ & $w+\frac{3}{2}$ & $w+2$ \\
$c$ & $-w$ & $-w+\frac{1}{2}$ & $-w+1$ & $-w+1$ & $-w+\frac{3}{2}$ & $-w+2$ \\
$\gamma_{5}$ & & + & & & + & \\
\hline
\end{tabular}

Table 2. Weyl $(w)$ and chiral $(c)$ weights of the chiral multiplet components. The chirality $\left(\gamma_{5}\right)$ of the fermion fields is also indicated.

$$
\begin{aligned}
\delta \Lambda_{i}= & -\frac{1}{2} \gamma^{a b} \not D F_{a b}^{-} \epsilon_{i}-\not D B_{i j} \varepsilon^{j k} \epsilon_{k}+C \varepsilon_{i j} \epsilon^{j}+\frac{1}{4}\left(\not D A \gamma^{a b} T_{a b i j}+w A \not D \gamma^{a b} T_{a b i j}\right) \varepsilon^{j k} \epsilon_{k} \\
& -3 \gamma_{a} \varepsilon^{j k} \epsilon_{k} \bar{\chi}_{[i} \gamma^{a} \Psi_{j]}-(1+w) B_{i j} \varepsilon^{j k} \eta_{k}+\frac{1}{2}(1-w) \gamma^{a b} F_{a b}^{-} \eta_{i} \\
\delta C= & -2 \varepsilon^{i j} \bar{\epsilon}_{i} \not D \Lambda_{j}-6 \bar{\epsilon}_{i} \chi_{j} \varepsilon^{i k} \varepsilon^{j l} B_{k l} \\
& -\frac{1}{4} \varepsilon^{i j} \varepsilon^{k l}\left((w-1) \bar{\epsilon}_{i} \gamma^{a b} \not D T_{a b j k} \Psi_{l}+\bar{\epsilon}_{i} \gamma^{a b} T_{a b j k} \not D \Psi_{l}\right)+2 w \varepsilon^{i j} \bar{\eta}_{i} \Lambda_{j}
\end{aligned}
$$

In the above equations, the derivatives $D$ are covariantized with respect to the gauge transformations of the superconformal algebra appropriate for each field, as shown in (B.2).

The vector multiplet is an example of a reduced chiral multiplet, with components:

$$
\mathcal{X}=\left(X, \Omega_{i}, W_{\mu}, Y_{i j}\right)
$$

where $X$ is a complex scalar, $\Omega_{i}$ is an $\mathrm{SU}(2)$ doublet of chiral fermions, $Y_{i j}$ is a real $\mathrm{SU}(2)$ triplet, and $W_{\mu}$ is a physical gauge field.

This multiplet is obtained from the chiral multiplet by imposing a specific constraint on the field representation. Specifically we impose a reality constraint on the complex $\mathrm{SU}(2)$ triplet $B_{i j}$, which alone fixes every component of the chiral multiplet in terms of the vector multiplet components. The identifications are as follows:

$$
\begin{aligned}
\left.A\right|_{\mathrm{vec}} & =X \\
\left.\psi_{i}\right|_{\mathrm{vec}} & =\Omega_{i} \\
\left.B_{i j}\right|_{\mathrm{vec}} & =Y_{i j}=\varepsilon_{i k} \varepsilon_{j l} Y^{k l}, \\
\left.F_{a b}^{-}\right|_{\mathrm{vec}} & =F_{a b}^{-}+\frac{1}{4}\left(\bar{\psi}_{\rho}^{i} \gamma_{a b} \gamma^{\rho} \Omega^{j}+\bar{X} \bar{\psi}_{\rho}^{i} \gamma^{\rho \sigma} \gamma_{a b} \psi_{\sigma}^{j}+\text { c.c }-\bar{X} T_{a b}{ }^{i j}\right) \epsilon_{i j}, \\
\left.\Lambda_{i}\right|_{\mathrm{vec}} & =-\varepsilon_{i j} \not D \Omega^{j} \\
\left.C\right|_{\mathrm{vec}} & =-2 \square_{\mathrm{c}} \bar{X}-\frac{1}{4} \hat{F}_{a b}^{+} T^{a b}{ }_{i j} \epsilon^{i j}-3 \bar{\chi}_{i} \Omega^{i},
\end{aligned}
$$

where the symbol $\square_{\mathrm{c}}=D^{\mu} D_{\mu}$ is the superconformal d'Alembertian and $F_{a b}$ is used to indicate the abelian field strength $F_{a b}=2 e_{a}^{[\mu} e_{b}{ }^{\nu]} \partial_{\mu} W_{\nu}$, which satisfies now a (superconformal) Bianchi identity.

In table 3 we show the Weyl and chiral weight assignment for the vector multiplet components. The Q- and S-supersymmetry transformation rules for the vector multiplet 


\begin{tabular}{|c|cccc|}
\hline & $X$ & $\Omega_{i}$ & $W_{\mu}$ & $Y_{i j}$ \\
\hline$w$ & 1 & $\frac{3}{2}$ & 0 & 2 \\
$c$ & -1 & $-\frac{1}{2}$ & 0 & 0 \\
$\gamma_{5}$ & & + & & \\
\hline
\end{tabular}

Table 3. Weyl $(w)$ and chiral $(c)$ weights of the vector multiplet components. The chirality $\left(\gamma_{5}\right)$ of the fermion field $\Omega_{i}$ is also indicated.

in a conformal background take the form,

$$
\begin{aligned}
\delta X & =\bar{\epsilon}^{i} \Omega_{i} \\
\delta \Omega_{i} & =2 \not D X \epsilon_{i}+\frac{1}{2} \varepsilon_{i j} \hat{F}_{\mu \nu} \gamma^{\mu \nu} \epsilon^{j}+Y_{i j} \epsilon^{j}+2 X \eta_{i}, \\
\delta W_{\mu} & =\varepsilon^{i j} \bar{\epsilon}_{i}\left(\gamma_{\mu} \Omega_{j}+2 \psi_{\mu j} X\right)+\varepsilon_{i j} \bar{\epsilon}^{i}\left(\gamma_{\mu} \Omega^{j}+2 \psi_{\mu}{ }^{j} \bar{X}\right), \\
\delta Y_{i j} & =2 \bar{\epsilon}_{(i} \not D \Omega_{j)}+2 \varepsilon_{i k} \varepsilon_{j l} \bar{\epsilon}^{(k} \not D \Omega^{l)} .
\end{aligned}
$$

The last multiplet we want to present is the covariant Weyl multiplet from which the Weyl squared multiplet is obtained. This is another reduced chiral multiplet, and its components read:

$$
\begin{aligned}
\left.A_{a b}\right|_{\mathbf{W}} & =T_{a b}{ }^{i j} \varepsilon_{i j}, \\
\left.\psi_{a b i}\right|_{\mathbf{w}} & =8 \varepsilon_{i j} R(Q)_{a b}^{j}, \\
\left.B_{a b i j}\right|_{\mathbf{W}} & =-8 \varepsilon_{k(i} R(\mathcal{V})_{a b j}^{-k}, \\
\left.\left(F_{a b}^{-}\right)^{c d}\right|_{\mathbf{W}} & =-8 \mathcal{R}(M)_{a b}^{-c d} \\
\left.\Lambda_{a b i}\right|_{\mathbf{W}} & =8\left(\mathcal{R}(S)_{a b i}^{-}+\frac{3}{4} \gamma_{a b} \not D \chi_{i}\right) \\
\left.C_{a b}\right|_{\mathbf{W}} & =4 D_{[a} D^{c} T_{b] c i j} \varepsilon^{i j}-\text { dual } .
\end{aligned}
$$

Note that the Weyl tensor is contained inside the highest independent component $\left(F_{a b}^{-}\right)^{c d}$ through the $\mathcal{R}(M)_{a b}^{-c d}$ curvature.

By squaring the covariant Weyl multiplet $\mathbf{W}$ a scalar chiral multiplet with $w=2$ is obtained,

$$
\begin{aligned}
A= & \left(T_{a b}{ }^{i j} \varepsilon_{i j}\right)^{2}, \\
\Psi_{i}= & 16 \varepsilon_{i j} R(Q)_{a b}^{j} T^{k l a b} \varepsilon_{k l}, \\
B_{i j}= & -16 \varepsilon_{k(i} R(\mathcal{V})^{k}{ }_{j) a b} T^{l m a b} \varepsilon_{l m}-64 \varepsilon_{i k} \varepsilon_{j l} \bar{R}(Q)_{a b}{ }^{k} R(Q)^{l a b}, \\
F^{-a b}= & -16 \mathcal{R}(M)_{c d}^{a b} T^{k l c d} \varepsilon_{k l}-16 \varepsilon_{i j} \bar{R}(Q)_{c d}^{i} \gamma^{a b} R(Q)^{c d j}, \\
\Lambda_{i}= & 32 \varepsilon_{i j} \gamma^{a b} R(Q)_{c d}^{j} \mathcal{R}(M)^{c d}{ }_{a b}+16\left(\mathcal{R}(S)_{a b i}+3 \gamma_{[a} D_{b]} \chi_{i}\right) T^{k l a b} \varepsilon_{k l} \\
& -64 R(\mathcal{V})_{a b}{ }_{i} \varepsilon_{k l} R(Q)^{a b l}, \\
C= & 64 \mathcal{R}(M)^{-c d}{ }_{a b} \mathcal{R}(M)_{c d}^{-a b}+32 R(\mathcal{V})^{-a b k}{ }_{l} R(\mathcal{V})_{a b}^{-l}{ }^{k} \\
& -32 T^{a b i j} D_{a} D^{c} T_{c b i j}+128 \overline{\mathcal{R}}(S)^{a b}{ }_{i} R(Q)_{a b}{ }^{i}+384 \bar{R}(Q)^{a b i} \gamma_{a} D_{b} \chi_{i} .
\end{aligned}
$$


Both the covariant Weyl multiplet $\mathbf{W}$ and its square are functions of the curvatures of the local superconformal algebra. As expected from a reduced chiral multiplet, the highest components of the Weyl multiplet are not independent.

Open Access. This article is distributed under the terms of the Creative Commons Attribution License (CC-BY 4.0), which permits any use, distribution and reproduction in any medium, provided the original author(s) and source are credited.

\section{References}

[1] D. Butter, B. de Wit, S.M. Kuzenko and I. Lodato, New higher-derivative invariants in $N=2$ supergravity and the Gauss-Bonnet term, JHEP 12 (2013) 062 [arXiv:1307.6546] [INSPIRE].

[2] R.M. Wald, Black hole entropy is the Noether charge, Phys. Rev. D 48 (1993) 3427 [gr-qc/9307038] [INSPIRE].

[3] A. Sen, Black hole entropy function and the attractor mechanism in higher derivative gravity, JHEP 09 (2005) 038 [hep-th/0506177] [INSPIRE].

[4] J.A. Harvey, R. Minasian and G.W. Moore, Non-Abelian tensor multiplet anomalies, JHEP 09 (1998) 004 [hep-th/9808060] [INSPIRE].

[5] P. Kraus and F. Larsen, Microscopic black hole entropy in theories with higher derivatives, JHEP 09 (2005) 034 [hep-th/0506176] [INSPIRE].

[6] P. Kraus and F. Larsen, Holographic gravitational anomalies, JHEP 01 (2006) 022 [hep-th/0508218] [INSPIRE].

[7] J.D. Brown and M. Henneaux, Central Charges in the Canonical Realization of Asymptotic Symmetries: An Example from Three-Dimensional Gravity, Commun. Math. Phys. 104 (1986) 207 [INSPIRE].

[8] B. Sahoo and A. Sen, Higher derivative corrections to non-supersymmetric extremal black holes in $N=2$ supergravity, JHEP 09 (2006) 029 [hep-th/0603149] [INSPIRE].

[9] D. Butter, B. de Wit and I. Lodato, Non-renormalization theorems and $N=2$ supersymmetric backgrounds, JHEP 03 (2014) 131 [arXiv:1401.6591] [INSPIRE].

[10] J.M. Maldacena, A. Strominger and E. Witten, Black hole entropy in M-theory, JHEP 12 (1997) 002 [hep-th/9711053] [INSPIRE].

[11] G. Lopes Cardoso, B. de Wit and T. Mohaupt, Corrections to macroscopic supersymmetric black hole entropy, Phys. Lett. B 451 (1999) 309 [hep-th/9812082] [INSPIRE].

[12] G. Lopes Cardoso, B. de Wit and T. Mohaupt, Deviations from the area law for supersymmetric black holes, Fortsch. Phys. 48 (2000) 49 [hep-th/9904005] [INSPIRE].

[13] E. Bergshoeff, M. de Roo and B. de Wit, Extended Conformal Supergravity, Nucl. Phys. B $182(1981) 173$.

[14] N. Banerjee, B. de Wit and S. Katmadas, The Off-Shell 4D/5D Connection, JHEP 03 (2012) 061 [arXiv:1112.5371] [INSPIRE].

[15] K. Hanaki, K. Ohashi and Y. Tachikawa, Supersymmetric Completion of an $R^{2}$ term in Five-dimensional Supergravity, Prog. Theor. Phys. 117 (2007) 533 [hep-th/0611329] [INSPIRE]. 
[16] D. Freed, J.A. Harvey, R. Minasian and G.W. Moore, Gravitational anomaly cancellation for M-theory five-branes, Adv. Theor. Math. Phys. 2 (1998) 601 [hep-th/9803205] [inSPIRE].

[17] B. Sahoo and A. Sen, BTZ black hole with Chern-Simons and higher derivative terms, JHEP 07 (2006) 008 [hep-th/0601228] [INSPIRE].

[18] P.H. Ginsparg, Applications of topological and differential geometric methods to anomalies in quantum field theory, in XVI GIFT International Seminar on Theoretical Physics: New Perspectives in Quantum Field Theory, Jaca (Huesca) Spain, 3-8 June 1985.

[19] J.A. Harvey, TASI 2003 lectures on anomalies, hep-th/0509097 [INSPIRE].

[20] A. Castro, J.L. Davis, P. Kraus and F. Larsen, 5D attractors with higher derivatives, JHEP 04 (2007) 091 [hep-th/0702072] [INSPIRE].

[21] K. Goldstein, N. Iizuka, R.P. Jena and S.P. Trivedi, Non-supersymmetric attractors, Phys. Rev. D 72 (2005) 124021 [hep-th/0507096] [INSPIRE].

[22] P.K. Tripathy and S.P. Trivedi, Non-supersymmetric attractors in string theory, JHEP 03 (2006) 022 [hep-th/0511117] [INSPIRE].

[23] A. Dabholkar, J. Gomes, S. Murthy and A. Sen, Supersymmetric Index from Black Hole Entropy, JHEP 04 (2011) 034 [arXiv: 1009.3226] [INSPIRE].

[24] M. Ozkan and Y. Pang, All off-shell $R^{2}$ invariants in five dimensional $\mathcal{N}=2$ supergravity, JHEP 08 (2013) 042 [arXiv: 1306.1540] [InSPIRE].

[25] M. Cvitan, P.D. Prester, S. Pallua and I. Smolic, Extremal black holes in D = 5: SUSY vs. Gauss-Bonnet corrections, JHEP 11 (2007) 043 [arXiv:0706.1167] [INSPIRE].

[26] M. Cvitan, P.D. Prester and A. Ficnar, $\alpha^{\prime 2}$-corrections to extremal dyonic black holes in heterotic string theory, JHEP 05 (2008) 063 [arXiv:0710.3886] [INSPIRE].

[27] B. de Wit, J.W. van Holten and A. Van Proeyen, Structure of $N=2$ Supergravity, Nucl. Phys. B 184 (1981) 77 [Erratum ibid. B 222 (1983) 516] [InSPIRE]. 